\title{
Enumerative Real Algebraic Geometry
}

\author{
Frank Sottile
}

Contents

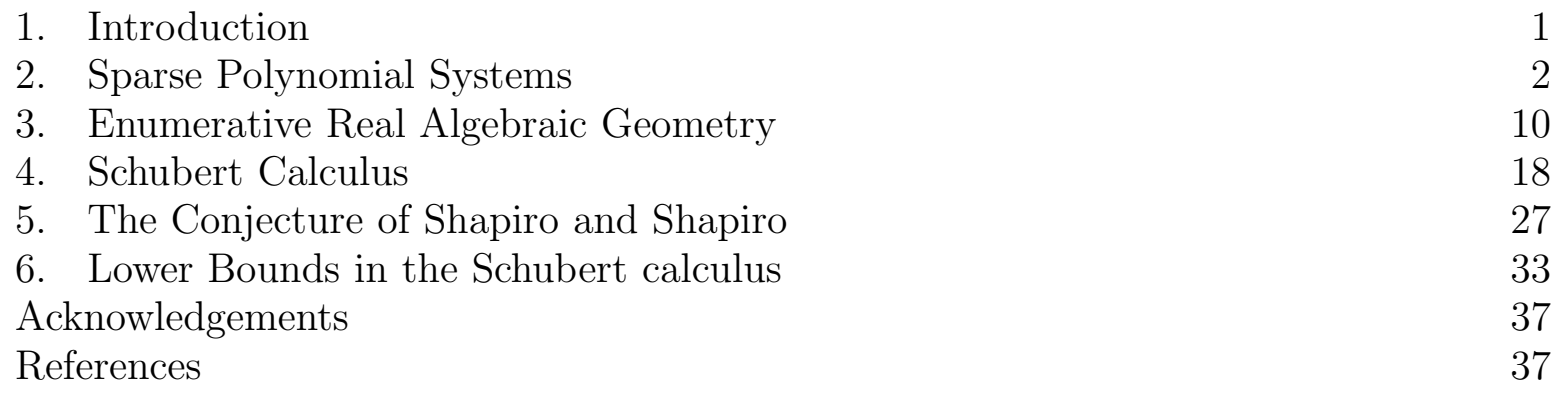

\section{Introduction}

Consider the following question.

Question 1.1. Find a priori information about the number of real solutions to a structured system of real polynomial equations

$$
0=f_{1}=f_{2}=\cdots=f_{N}, \quad \text { where each } f_{i} \in \mathbb{R}\left[x_{1}, \ldots, x_{n}\right] .
$$

Two well-defined classes of structured polynomial systems have been studied from this point of view - sparse systems, where the structure is encoded by the monomials in the polynomials $f_{i}$-and geometric systems, where the structure comes from geometry. This second class consists of polynomial formulations of enumerative geometric problems, and in this case Question 1.1 is the motivating question of enumerative real algebraic geometry, the subject of this survey. Treating both sparse polynomial systems and enumerative geometry together in the context of Question 1.1 gives useful insight.

Given a system of polynomial equations (1.1) with $d$ complex solutions, we know the following easy facts about its number $\rho$ of real solutions.

We have $\rho \leq d$, and

since $d-\rho$ an even integer, $\rho \geq \begin{cases}0 & \text { if } d \text { is even } \\ 1 & \text { if } d \text { is odd . }\end{cases}$

In a surprising number of cases, much better information than this is known.

3 June 2001.

Research Supported in part by NSF grant DMS-0070494.

2000 Mathematics Subject Classification. 14P99, 12D10, 14N10, 14N15, 14M15, 14M25, 14M17. 
Structured systems occur in families of systems sharing the same structure. The common structure determines the number $d$ of complex solutions to a general member of the family. We assume throughout that a general system in any family has only simple solutions in that each complex solution occurs without multiplicity.

Given such a family of structured systems whose general member has $d$ complex solutions, perhaps the ultimate answer to our motivating question is to determine exactly which numbers $\rho$ of real solutions can occur and also which systems have a given number of real solutions. Because this level of knowledge may be unattainable, we will be satisfied with less knowledge.

For example, are the trivial bounds given in (1.2) sharp? That is, do there exist systems attaining the maximal and minimal number of real solutions allowed by (1.2)? If these bounds are not sharp, do there exist better sharp bounds? Perhaps we are unable to determine sharp bounds, but can exhibit systems in a family with many (or few) real solutions. This gives lower bounds on the maximum number of real solutions to a system in a family (or upper bounds on the minimum number).

These answers have two parts: bounds and constructions. We shall see that bounds (or other limitations) often come from topological considerations. On the other hand, the constructions often come by deformations from/to a degenerate situation. In enumerative geometry, this is the classical technique of special position, while for sparse systems, it is Viro's method of toric deformations.

In Section 2 we discuss sparse polynomial systems from the point of view of Question 1.1. The heart of this survey begins in Section 3, where we discuss some of the myriad examples of enumerative geometric problems that have been studied from this perspective. In particular, for many enumerative problems the upper bound (1.2) is sharp. In Section 4, we concentrate on enumerative problems from the Schubert calculus, where much work has been done on this question of real solutions. Section 5 is devoted to a conjecture of Shapiro and Shapiro, whose study has led to many recent results in this area. Finally, in Section 6 we describe new ideas of Eremenko and Gabrielov giving lower bounds better than (1.2) for many enumerative problems.

\section{Sparse Polynomial Systems}

Perhaps the most obvious structure of a system of $n$ polynomials in $n$ variables

$$
f_{1}\left(x_{1}, \ldots, x_{n}\right)=f_{2}\left(x_{1}, \ldots, x_{n}\right)=\cdots=f_{n}\left(x_{1}, \ldots, x_{n}\right)=0,
$$

is the list of total degrees of the polynomials $f_{1}, f_{2}, \ldots, f_{n}$. For such a system, we have the degree or Bézout upper bound, which is a consequence of the refined Bézout Theorem of Fulton and MacPherson [20, §1.23].

Theorem 2.1 (Bézout Bound). The system (2.1), where the polynomial $f_{i}$ has total degree $d_{i}:=\operatorname{deg}\left(f_{i}\right)$, has at most $d_{1} \cdot d_{2} \cdots d_{n}$ isolated complex solutions.

The Bézout bound on the number of real solutions is sharp. For example, if

$$
f_{i}:=\left(x_{i}-1\right)\left(x_{i}-2\right) \cdots\left(x_{i}-d_{i}\right) \quad i=1, \ldots, n,
$$

then the system (2.1) has $d_{1} \cdot d_{2} \cdots d_{n}$ real solutions. The reader is invited to construct systems with the minimum possible number (0 or 1 ) of real solutions.

A system of polynomial equations with only simple solutions, but with fewer solutions than the Bézout bound is called deficient. For example, fewer monomials in the polynomials lead to fewer solutions. We make this idea more precise. A monomial (or rather its 
exponent vector) is a point of $\mathbb{N}^{n}$. The convex hull of the monomials in $f$ is its Newton polytope, New $(f)$, a polytope with integral vertices. The terms of $f$ are indexed by the lattice points, $N e w(f) \cap \mathbb{N}^{n}$, in the Newton polytope of $f$.

Figure 1 displays the monomials (dots) and Newton polytopes of the polynomials

$$
\begin{aligned}
& f=1+x-y+x y z^{4}, \\
& g=1+x-y+3 z-z^{3}+2 z^{4} .
\end{aligned}
$$
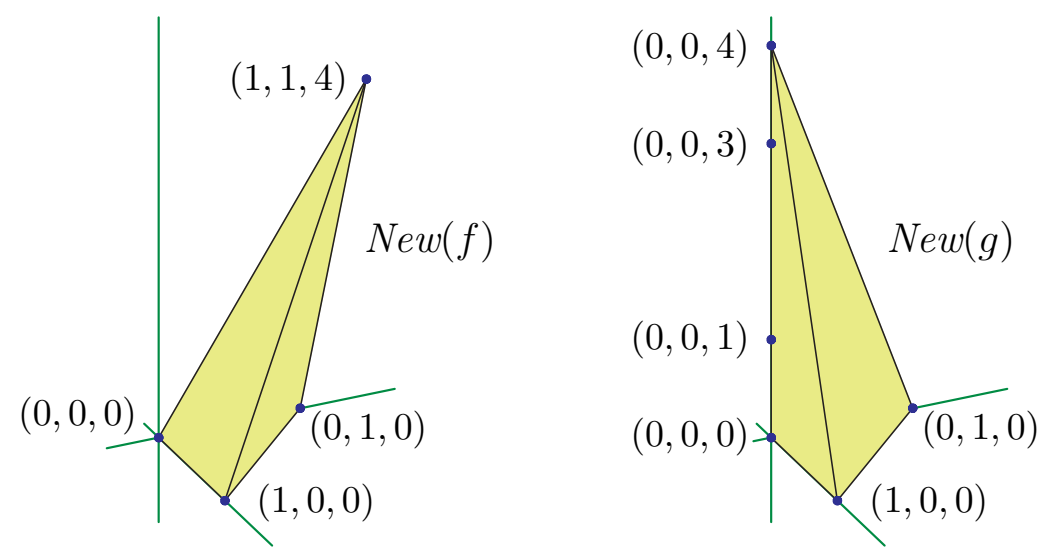

Figure 1. Newton Polytopes

Given polytopes $P_{1}, P_{2}, \ldots, P_{m} \subset \mathbb{R}^{n}$, their Minkowski sum is the pointwise sum

$$
P_{1}+P_{2}+\cdots+P_{m}=\left\{p_{1}+p_{2}+\cdots+p_{m} \mid p_{i} \in P_{i}, i=1,2, \ldots, m\right\} .
$$

The mixed volume, $M V\left(P_{1}, P_{2}, \ldots, P_{m}\right)$, of a collection of $m$ polytopes is

$$
\frac{\partial}{\partial t_{1}} \frac{\partial}{\partial t_{2}} \cdots \frac{\partial}{\partial t_{m}}\left[\text { Volume of } t_{1} P_{1}+t_{2} P_{2}+\cdots+t_{m} P_{m}\right]
$$

When there are $n$ equal polytopes, the mixed volume is $n ! \operatorname{Vol}(P)$, the normalized volume of the common Newton polytope $P$, and when each $P_{i}$ is a line segment of length $d_{i}$ in the $i$ th coordinate (so that $P_{i}=\operatorname{New}\left(f_{i}\right)$, where $f_{i}$ is the polynomial given in (2.2)), the mixed volume is the Bézout number $d_{1} d_{2} \cdots d_{n}$.

Given a list $\left(P_{1}, P_{2}, \ldots, P_{n}\right)$ of polytopes in $\mathbb{R}^{n}$ with vertices in the integral lattice $\mathbb{N}^{n}$, a sparse polynomial system with this structure is a system of polynomials (2.1) with $\operatorname{New}\left(f_{i}\right)=P_{i}$. These sparse systems may have trivial solutions where some coordinates vanish. Thus we only consider solutions in $\left(\mathbb{C}^{\times}\right)^{n}$. We have the following basic result on the number of solutions to such a sparse system of polynomials.

TheOREM 2.2 (BKK bound). A sparse polynomial system (2.1) with structure $\left(P_{1}, P_{2}, \ldots, P_{n}\right)$ has at most $M V\left(P_{1}, P_{2}, \ldots, P_{n}\right)$ isolated solutions in $\left(\mathbb{C}^{\times}\right)^{n}$. If the system is generic given its structure, then it has exactly this number of solutions in $\left(\mathbb{C}^{\times}\right)^{n}$.

This result was developed in a series of papers by Kouchnirenko [45], Bernstein [1], and Khovanskii [40]. For simplicity of exposition, we will largely restrict ourselves to the case when the polynomials all have the same Newton polytope $P$.

Given a polytope $P$ with vertices in the integral lattice, what are the possible numbers of real solutions to systems with that Newton polytope? We shall focus on understanding 
$\rho(P)$, the maximum number of real solutions to a sparse system with Newton polytope $P$. The following example serves as an introduction to this question.

EXAMPLE 2.3. Let $d$ be a positive integer, and set

$$
\begin{aligned}
& P_{d}:=\text { Convex hull }\{(0,0,0),(1,0,0),(0,1,0),(1,1, d)\}, \text { and } \\
& Q_{d}:=\text { Convex hull }\{(0,0,0),(1,0,0),(0,1,0),(0,0, d)\} .
\end{aligned}
$$

(Figure 1 shows $P_{4}$ and $Q_{4}$.) These tetrahedrons each have normalized volume $d$.

A general sparse system with Newton polytope $P_{d}$

$$
\begin{aligned}
& A_{1} x y z^{d}+B_{1} x+C_{1} y+D_{1}=0 \\
& A_{2} x y z^{d}+B_{2} x+C_{2} y+D_{2}=0 \\
& A_{3} x y z^{d}+B_{3} x+C_{3} y+D_{3}=0
\end{aligned}
$$

is equivalent to a system of the form

$$
z^{d}=\alpha, \quad x=\beta, \quad y=\gamma,
$$

where $\alpha, \beta, \gamma \in \mathbb{R}$. Thus the original system has $d$ complex solutions, but only 0 , 1 , or 2 real solutions, so $\rho\left(P_{d}\right)$ is 1 or 2 , depending upon the parity of $d$.

A general sparse system with Newton polytope $Q_{d}$ is 3 polynomials of the form

$$
A x+B y+C(z),
$$

where $C(z)$ has degree $d$. This is equivalent to a system of the form

$$
f(z)=0, \quad x=g(z), \quad y=h(z),
$$

where $f(z), g(z)$, and $h(z)$ are real polynomials with $\operatorname{deg}(f)=d$ but $\operatorname{deg}(g)=\operatorname{deg}(h)=$ $d-1$. In this case, the general system again has $d$ complex solutions, but there are systems with any number of real solutions, so $\rho\left(Q_{d}\right)=d$.

2.1. Polyhedral homotopy algorithm. The polyhedral homotopy algorithm of Huber and Sturmfels [33] deforms the sparse system (2.1) into a system where the number of solutions is evident. It gives an effective demonstration of the BKK bound and is based upon Sturmfels's generalization [82] of Viro's method for constructing real varieties with controlled topology [85].

ExAmple 2.4. Suppose $P$ is a $n$-simplex which meets the integral lattice only at its vertices. Translating one vertex to the origin, the others are linearly independent. (Translating corresponds to division by a monomial.) Let $M$ be the $n \times n$ integral matrix whose columns are these vertices. Multiplying $M$ by an invertible integral matrix and taking the resulting columns to be a basis for $\mathbb{R}^{n}$ corresponds to a multiplicative change of coordinates

$$
\left(x_{1}, x_{2}, \ldots, x_{n}\right) \longmapsto\left(y^{m_{1}}, y^{m_{2}}, \ldots, y^{m_{n}}\right),
$$

with $y_{1}, y_{2}, \ldots, y_{n} \in\left(\mathbb{C}^{\times}\right)^{n}$ and $m_{1}, m_{2}, \ldots, m_{n}$ linearily independent integer vectors. Doing this for the Smith normal form of $M$ transforms a polynomial $f$ with Newton polytope $P$ into a polynomial of the form

$$
c_{0}+c_{1} y_{1}^{d_{1}}+c_{2} y_{2}^{d_{2}}+\cdots+c_{n} y_{n}^{d_{n}}
$$


where $d_{1}\left|d_{2}\right| \cdots \mid d_{n}$ and $d_{1} d_{2} \cdots d_{n}=n ! \operatorname{Vol}(P)$, the normalized volume of $P$. (These $d_{i}$ are the invariant factors of the integral matrix $M$.) A system consisting of $n$ general polynomials of the form (2.3) is equivalent to one of the form

$$
y_{1}^{d_{1}}=\alpha_{1}, \quad y_{2}^{d_{2}}=\alpha_{2}, \quad \ldots, \quad y_{2}^{d_{n}}=\alpha_{n} .
$$

Thus a general system whose Newton polytope is $P$ has $n ! \operatorname{Vol}(P)$ simple complex solutions.

The combinatorial structure underlying the homotopy algorithm of Huber and Sturmfels is that of a regular triangulation, which is a special case of a regular subdivision. A regular subdivision $P_{w}$ of a lattice polytope $P$ is given by a lifting function

$$
w: P \cap \mathbb{N}^{n} \longrightarrow \mathbb{Q} \geq 0
$$

as follows. Set

$$
Q:=\text { Convex hull }\left\{(a, w(a)) \mid a \in P \cap \mathbb{N}^{n}\right\} \subset \mathbb{R}^{n+1}
$$

This lifted polytope $Q$ has distinguished lower facets, those facets whose inward pointing normal vector has positive last coordinate. Forgetting the last coordinate projects these lower facets into $\mathbb{R}^{n}$ (and hence $P$ ). Their totality gives the regular subdivision $P_{w}$ of $P$. When all the lattice points in $P \cap \mathbb{N}^{n}$ lift to vertices of lower facets of $Q$, and these lower facets are all simplices, then $P_{w}$ is a regular triangulation of $P$.

In Figure 2 the triangulation on the left is regular and the triangulation on the right is not regular. Consider a hypothetical lifting function $w$ for the triangulation on the right. We assume that $w$ takes the value 0 at the three interior vertices. The clockwise neighbour of any vertex of the big triangle must be lifted higher than that vertex. (Consider the figure they form with the parallel edge of the interior triangle.) Since the edge of the big triangle is lifted to a concave broken path, this implies that each vertex is lower than its clockwise neighbour, which is impossible, except in some M.C. Escher woodcuts.
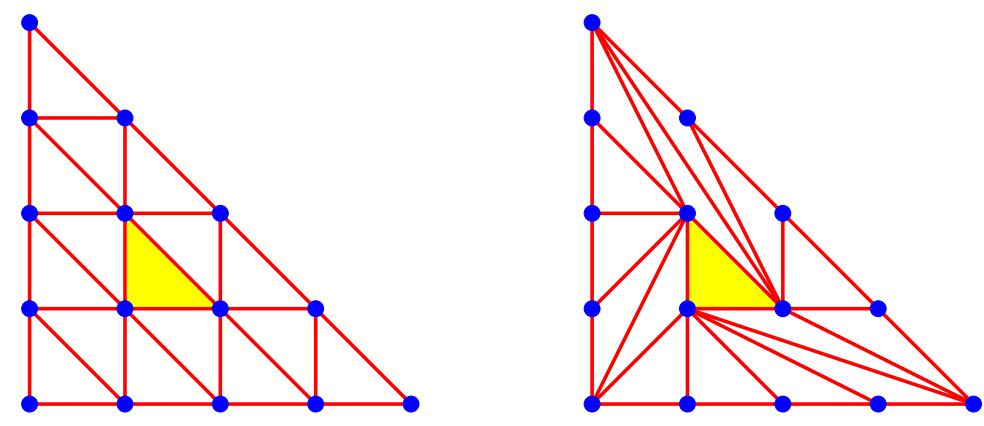

FiguRE 2. Regular and non-regular triangulations

Crucial to this algorithm are polynomial systems derived from the original system and the regular subdivision $P_{w}$ of $P$ by a lifting function $w$. Given a polynomial $f$ with Newton polytope $P$ and a face $F$ of the subdivision $P_{w}$, consider the sum of terms of $f$ with exponent vector in $F$, restricting $f$ to the face $F$. Doing this for each polynomial $f_{i}$ in our original system, we obtain the facial subsystem of (2.1) given by $F$.

We continue with the algorithm of Huber and Sturmfels. Given a lifting function $w: P \cap \mathbb{N}^{n} \rightarrow \mathbb{Q}_{\geq 0}$ and a polynomial $f$ with Newton polytope $P$

$$
f(x)=\sum_{a \in P \cap \mathbb{N}^{n}} c_{a} x^{a},
$$


we multiply the term with exponent $a$ by $t^{w(a)}$ to obtain

$$
f(x ; t):=\sum_{a \in P \cap \mathbb{N}^{n}} c_{a} x^{a} t^{w(a)} .
$$

Modifying all polynomials in the original sparse system in this way gives the lifted system, a family of sparse systems depending upon the parameter $t$.

$$
f_{1}(x ; t)=f_{2}(x ; t)=\cdots=f_{n}(x ; t)=0,
$$

Solutions to this system are algebraic functions $t \mapsto x(t)$ in the parameter $t$. In a neighborhood of 0 in the complex plane, each branch is expressed as a Puiseaux series

$$
\begin{aligned}
x(t) & =\left(x_{1}(t), x_{2}(t), \ldots, x_{n}(t)\right), \quad \text { with } \\
x_{i}(t) & =t^{u_{i}} y_{i}+\text { higher order terms in } t,
\end{aligned}
$$

where the $y_{i}$ are non-zero constants and exponents $u_{i} \in \mathbb{Q}$. Substituting this expression into (2.4), we obtain

$$
0=\sum_{a \in P \cap \mathbb{N}^{n}} c_{a} y^{a} t^{u \cdot a+w(a)}+\text { higher order terms in } t .
$$

The exponent of $t$ is the dot product $(u, 1) \cdot(a, w(a))$. Thus the terms of lowest order in $t$ correspond to points $(a, w(a))$ in the lifted polytope where the exponent vector $(u, 1)$ achieves its minimum - a face in the lower hull of the lifted polytope. Let $F$ be the corresponding face of the regular subdivision $P_{w}$. Removing the common factor $t^{u \cdot a+w(a)}$ from these lowest order terms gives the restriction of $f$ to the face $F$. Thus the coefficients $y_{i}$ of the leading terms of the Puiseaux expansion are solutions to the facial subsystem of the original system (2.1) given by the face $F$ selected by the initial Puiseaux exponents.

Suppose the original system is general in the sense that each of its facial subsystems given by faces $F$ of the regular subdivision has solutions only if $\operatorname{Vol}(F)>0$. Consider substituting the Puiseaux series into the polynomial system (2.4) and then taking the limit as $t$ approaches 0 . The resulting polynomial system for the leading coefficients $y$ of the Puiseaux series are solutions to the corresponding facial system. By previous assumption, this has no solutions unless $F$ is a facet of the subdivision $P_{w}$. In that case, the inward pointing normal vector $(u, 1)$ to the corresponding facet of the lifted polytope gives the initial exponent $u$ of the Puiseaux expansion.

The full Puiseaux series can be reconstructed from its initial terms (see [33 for details) giving the 1-1 correspondence

$$
\left\{\begin{array}{l}
\text { Solutions to facial subsystems } \\
\text { of }(2.1) \text { given by facets of } P_{w}
\end{array}\right\} \Longleftrightarrow\left\{\begin{array}{l}
\text { Branches of the algebraic } \\
\text { function } t \mapsto x(t) \text { near } t=0
\end{array}\right\}
$$

This is the number of solutions to (2.4) for general $t \in \mathbb{C}$, so the number of solutions to the original sparse system equals the number of solutions to all the facial subsystems given by facets of $P_{w}$.

Now suppose the regular subdivision $P_{w}$ is a regular triangulation and each facial subsystem is general. Then the facets $F$ of $P_{w}$ are simplices with no interior vertices. Since general sparse systems whose Newton polytope is such a simplex have exactly $n ! \operatorname{Vol}(F)$ solutions, the number of solutions to facial subsystems given by facets of $P_{w}$ is exactly the sum of the normalized volumes of all facets of $P_{w}$, which is the normalized volume of $P$. 
2.2. Real solutions to sparse polynomial systems. How many real solutions are there to a sparse polynomial system (2.1) with common Newton polytope $P$ ? By Example 2.4, if $P$ is the convex hull of $v_{0}, v_{1}, \ldots, v_{n}$, a simplex with no interior lattice points, then a general system (2.1) is equivalent to the system of binomials

$$
y_{1}^{d_{1}}=\alpha_{1}, \quad y_{2}^{d_{2}}=\alpha_{2}, \quad \ldots, \quad y_{n}^{d_{n}}=\alpha_{n},
$$

with each $a_{i} \neq 0$. Here, $d_{1}\left|d_{2}\right| \cdots \mid d_{n}$ are the invariant factors of the matrix whose $i$ th column is $v_{i}-v_{0}$ and $d_{1} d_{2} \cdots d_{n}$ is the normalized volume of $P$. Following Sturmfels 81], let $e(P)$ be the number of these invariant factors which are even. If $e(P)=0$, so that $d$ is odd, then $P$ is an odd cell.

Proposition 2.5. The polynomial system (2.5) has $2^{e(P)}$ real solutions if $a_{i}>0$ whenever $d_{i}$ is even, and 0 real solutions otherwise. In particular, if $P$ is an odd cell, then there is one real solution.

Theorem 2.6 (Sturmfels [81, Corollary 2.3]). The maximum number $\rho(P)$ of real solutions to a sparse polynomial system (2.1) with common Newton polytope $P$ satisfies

$$
\text { \# odd cells in } P_{w} \leq \rho(P),
$$

for any regular triangulation $P_{w}$ of $P$.

ProOF. In the limit as $t \rightarrow 0$, the lifted system (2.4) given by a regular triangulation $P_{w}$ of $P$ becomes the disjunction of facial subsystems of (2.1), one for each facet $F$ of $P_{w}$. Thus the number of real solutions in the limit is a constructive lower bound for $\rho(P)$.

By Proposition 2.5, the number of odd cells in $P_{w}$ is a lower bound on the number of real solutions to the facial subsystem given by the facets of $P_{w}$.

Proposition 2.5 also gives an upper bound on the limiting number of real solutions $\rho$ of the lifted system (2.4) as $t \rightarrow 0$ (due to Sturmfels [81, Theorem 2.2].)

$$
\rho \leq \sum_{F \text { a facet of } P_{w}} 2^{e(F)}
$$

More sophisticated accounting of the possible signs of the coefficients of facial subsystems improves this bound. This accounting is accomplished in [56] and [37, leading to a combinatorial upper bound for such limiting systems. Itenberg and Roy [37] show there is a system (2.1) for which this upper bound is attained, and thus obtain

$$
\text { Combinatorial upper bound for limiting systems } \leq \rho(P) \text {. }
$$

Itenberg and Roy then conjectured that this combinatorial bound was in fact the global bound, that is, they conjectured that the maximal number of real solutions occurs in limiting systems. They also gave a similar bound for $\rho_{+}(P)$, the number of solutions with positive coordinates. This was too optimistic, for Li and Wang [50] found a remarkably simple counterexample to this conjecture of Itenberg and Roy

$$
y-x-1=200-100 y^{3}+900 x^{3}-x^{3} y^{3}=0 .
$$

This system has 3 positive solutions

$$
(0.317659,1.317659), \quad(.659995,1.659995), \quad \text { and }(8.12058,9.12058),
$$

but the combinatorial upper bound is 2 . Thus systems with the maximal number of real solutions cannot in general be constructed with these limiting techniques.

We still have more questions than answers. Among the questions are: 
1. Improve this lower bound for $\rho(P)$ (or for $\rho_{+}(P)$ ) of Itenberg and Roy.

2. Find new general methods to construct systems with many real solutions.

3. Determine which polytopes $P$ have the property that $\rho(P)<n ! \operatorname{Vol}(P)$, that is, not all the solutions can be real.

2.3. Fewnomial bounds. These definitions, constructions, and results apply to what have come to be known as fewnomial systems. A fewnomial is a polynomial $f$ with few monomials - the monomials of $f$ are members of some set $\mathcal{A}$ not necessarily equal to the lattice points within its convex hull. In particular, the results of Section 2.2 give lower bounds on the maximum number of real solutions $\rho(\mathcal{A})$ to a system of fewnomials whose monomials are from $\mathcal{A}$. Here, we use a regular triangulation $\Delta_{w}$ of the point set $\mathcal{A}$ induced from a lifting function $w: \mathcal{A} \rightarrow \mathbb{Q}$.

When $n=1$, consider the binomial (a fewnomial) system

$$
x^{d}-1=0 .
$$

This has $d$ complex solutions. We similarly expect that the number of complex solutions to a fewnomial system is equal to the BKK bound. The above binomial system has either 1 or 2 real solutions, and so the number of real solutions to a fewnomial system should be less than the BKK bound. The question is: How much less?

Khovanskii 40, 41 established a very general result concerning systems where each $f_{i}$ is a polynomial function of the monomials $x^{a}$ for $a \in \mathcal{A}$. He proves that the number of real solutions to such a system is at most $2^{n} 2^{\left(\begin{array}{c}N \\ 2\end{array}\right)}(n+1)^{N}$, where $N=\# \mathcal{A}$, the number of monomials in $\mathcal{A}$. When the polynomial functions are linear, they are polynomials with monomials from $\mathcal{A}$, and hence we have Khovanskii's fewnomial bound.

$$
\rho(\mathcal{A}) \leq 2^{n} \cdot 2^{\left(\begin{array}{l}
N \\
2
\end{array}\right)}(n+1)^{N}
$$

While this bound seems outrageously large, it does not depend upon the volume of the convex hull of $\mathcal{A}$, but rather on the algebraic complexity - the ambient dimension $n$ and the size $N$ of $\mathcal{A}$. That such a bound exists at all was revolutionary.

We compare this complexity bound to the combinatorial upper bound (2.6) on the number of real solutions to a lifted fewnomial system (2.4) in the limit as $t \rightarrow 0$. The invariant $e(F)$ of a facet of the regular triangulation $\Delta_{w}$ of $\mathcal{A}$ is at most $n$. Thus

$$
\rho \leq 2^{n} \cdot \# \text { facets of } \Delta_{w}\left(\leq 2^{n} \cdot\left(\begin{array}{c}
N \\
n+1
\end{array}\right)\right)
$$

as a facet of $\Delta_{w}$ involves $n+1$ points of $\mathcal{A}$. This bound is typically much lower than Khovanskii's bound. For example, consider two trinomials in two variables. Here $n=2$, and after multiplying each equation by a suitable monomial, we have $\# \mathcal{A}=5$. Thus we have Khovanskii's fewnomial bound

$$
\rho(\mathcal{A}) \leq 2^{2} \cdot 2^{\left(\begin{array}{l}
5 \\
2
\end{array}\right)} \cdot 3^{5}=995,328
$$


In contrast, a triangulation of 5 points in the plane has at most 5 simplices

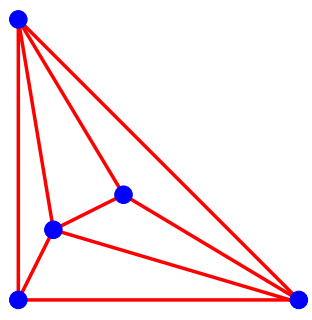

and so the bound $\rho$ for limiting lifted systems is

$$
\rho \leq 2^{2} \cdot 5=20 \text {. }
$$

It may be more feasible to look for bounds on $\rho_{+}(\mathcal{A})$, the number of solutions with positive coordinates. Kouchnirenko made ${ }^{\dagger}$ the following conjecture.

ConjeCture 2.7 (Kouchnirenko (see [5, p. 300])). A system of $n$ polynomials in $n$ variables with only simple solutions whose ith polynomial has $m_{i}$ monomials has at most

$$
\left(m_{1}-1\right)\left(m_{2}-1\right) \cdots\left(m_{n}-1\right)
$$

solutions with positive coordinates.

For a system of two trinomials in 2 variables, Kouchnirenko's conjecture asserts that $\rho_{+} \leq 4$. In 2000, Haas [26] gave the counterexample

$$
\begin{aligned}
& x^{108}+1.1 y^{54}-1.1 y=0 \\
& y^{108}+1.1 x^{54}-1.1 x=0
\end{aligned}
$$

a system of two trinomials with 5 solutions having positive coordinates. Although Kouchnirenko's conjecture is false, the question remains: Is the true value for $\rho_{+}(\mathcal{A})$ (or $\rho(\mathcal{A})$ ) closer to Khovanskii's bound or to the number in Kouchnirenko's conjecture? Recent work suggests that it is the latter.

Theorem 2.8 (Li, Rojas, and Wang [49]). A system of 2 polynomials in 2 variables

$$
f_{1}(x, y)=f_{2}(x, y)=0,
$$

where $f_{1}$ has 3 terms and $f_{2}$ has $m$ terms, and every solution is simple, has at most

$$
2 \cdot\left(2^{m-1}-1\right)
$$

solutions with positive coordinates.

When $m=3$, so we have two trinomials in 2 variables, this gives a bound of 6 . There is presently no known example with more that 5 positive solutions. Does Theorem 2.8 give the correct value of 6 , or is Haas's construction with 5 solutions the best possible?

REMARK 2.9. Khovanskii's bound for the number of real solutions with positive coordinates holds also for systems of power functions, where the exponents of monomials are arbitrary real numbers. One might suppose that this added generality is the source of the large size of his bound and its apparent lack of tightness for polynomial systems. However, Napoletani [55] has shown that the complexity bounds are the same for both polynomials and for power functions.

\footnotetext{
${ }^{\dagger}$ Apparently, Kouchnirenko did not believe this bound. Nonetheless, the conjecture and its attribution have passed into folklore.
} 


\section{Enumerative Real Algebraic Geometry}

In his treatise on enumerative geometry, Schubert 63 declared enumerative geometry to be concerned with all questions of the following form: How many geometric figures of a fixed type satisfy certain conditions? This includes problems as diverse as the number of lines on a cubic surface (27) and the number of lines meeting four fixed lines in 3-space (see Section 4 for the answer). These are archetypes for two distinct classes of enumerative geometric problems. For the purpose of this survey, we ignore the first class (except in Section 3.1) and concentrate on the second. Specifically, we consider conditions imposed by geometric figures that may be moved independently into general position.

Enumerative geometry (in the broad sense) had a great flourishing in the 19th century in the hands of Chasles, Schubert, Halphen, Zeuthen, and others. (The survey of Kleiman 43 is a good historical source.) At that time, it had long been recognized that it was necessary to work over the complex numbers to ensure the existence of solutions. We know of only a handful of cases where the number of real solutions was considered. (We discuss some in Section 3.1.) Asking how many solutions can be real is the motivating question of enumerative real algebraic geometry and an analog of Question 1.1.

QUESTION 3.1. In a given enumerative geometric problem, if the general figures are chosen to be real, how many of the solution figures can be real?

For example, how many of the lines on a real cubic surface can be real? (Answer: all 27.) How many of the lines meeting four given real lines can be real? (Answer: all can.) This question was posed by Fulton [21, p. 55]: "The question of how many solutions of real equations can be real is still very much open, particularly for enumerative problems."

This problem is fundamentally hard. Of the geometric figures satisfying real conditions, some will be real while the rest will occur in complex conjugate pairs, and the number which are real will depend subtly on the configuration of the conditions. Despite this difficulty, this is an important question often asked in applications.

One fruitful variant has been whether it is possible that all solution figures can be real. We call an enumerative problem fully real if there are general real conditions for which all solution figures are real. That is, if the upper bound of $(1.2)$ is sharp. In light of the situation for sparse polynomial systems, it is quite surprising that there are no known enumerative problems which are not fully real. For this it is important that the conditions are imposed by general figures. A related question is whether the opposite situation can occur: Is it possible to have no (or only one) real solutions? We give some examples in Section 4.2.4. We shall see in Section 6 that there are many enumerative problems whose number of real solutions is always at least 2 .

In the above passage, Fulton [21] goes on to ask: "For example, how many of the 3264 conics tangent to five general conics can be real?" He answered this question in the affirmative in 1986, but did not publish that result. Later, Ronga, Tognoli, and Vust [61] gave a careful argument that all 3264 can be real. This example is very striking, both for the number, 3264, and because this problem of conics has long been an important testing ground for ideas in enumerative geometry.

One difficulty with enumerative real algebraic geometry is that there are few techniques or theorems with a wide range of applicability. Sometimes a direct calculation suffices (Sections 3.3 and 3.4) or more commonly real solutions are constructed by deforming from a special configuration, as in the homotopy algorithm of Huber and Sturmfels in Section 2.1. Attempts to formalize this method include 'Schubert induction' [75]; this is 
presented in the proof of Theorem 4.6, and used to establish the other results of Section 4 . Another formalization is the notion of 'real effective algebraic equivalence' [70]. That (together with Theorem 4.4) gives such results as [70, Theorem 18]: The enumerative problem of $\left(\begin{array}{c}2 n-2 \\ n-1\end{array}\right) n^{2 n-3}$ codimension 2 planes meeting $2 n-2$ rational normal curves is fully real and [68, Corollary 5]: The enumerative problem of 11,010,048 2-planes in $\mathbb{P}^{5}$ meeting 9 general Veronese surfaces is fully real.

We devote the rest of this section to a description of some enumerative problems in the context of Question 3.1.

3.1. Enumerative problems not involving general conditions. We give a brief tour of some enumerative problems that do not involve general conditions, but nonetheless raise some interesting questions regarding real solutions.

Schläfli (see the survey of Coxeter [11]) showed there are 4 possibilities for the number of real lines on a real cubic surface: $27,15,7$, or 3 . Recent developments in enumerative geometry (mirror symmetry) have led to the solution of a large class of similar enumerative problems involving, among other things, the number of rational curves of a fixed degree on a Calabi-Yau threefold. (See the book of Cox and Katz [10].) For example, on a general quintic hypersurface in $\mathbb{P}^{4}$ there are 2875 lines [28], 609,250 conics [38, and 371,206,375 twisted cubics. The number of twisted cubics and higher degree rational curves was computed in the seminal paper of Candelas, de la Ossa, Green, and Parkes [6]. How many of the curves can be real in problems of this type? For example, how many real lines can there be on a real quintic hypersurface in $\mathbb{P}^{4}$ ?

A real homogeneous polynomial $f(x)$ is positive semi-definite (psd) if $f(x) \geq 0$ whenever $x$ is real. Hilbert [29] proved that a psd ternary quartic is a sum of three squares of real quadratic forms. In fact, a general quartic is a sum of three squares of complex quadratic forms in 63 different ways [86. Powers and Reznick [58] studied the question of how many ways one may represent a ternary quartic as a real sum or difference of three squares. In every instance, they found that 15 of the 63 ways involved real quadratic forms. Is it true that a general psd quartic is a sum or a differrence of three real squares in exactly 15 different ways? ${ }^{\dagger}$ In how many ways is it a sum of three squares?

A general plane curve $C$ of degree $d$ has $3 d(d-2)$ flexes. These are the points on $C$ where the Hessian determinant of the form defining $C$ vanishes. Since the Hessian determinant has degree $3(d-2)$, we expect there to be $3 d(d-2)$ such points. This involves intersecting the curve with its Hessian curve, and not with a general curve of degree $3(d-2)$. A real smooth plane cubic has 3 of its 9 flexes real. Zeuthen [89] found that at most 8 of the 24 flexes of a real plane quartic can be real. An example of a plane quartic with 8 real flexes is provided by the Hilbert quartic [30], which is defined by

$$
\left(x^{2}+2 y^{2}-z^{2}\right)\left(2 x^{2}+y^{2}-z^{2}\right)+z^{4} / 100=0 .
$$

We display this curve in Figure 3, marking the flexes with dots. Klein 44 later showed that a general real plane curve has at most $1 / 3$ of its flexes real.

Harnack [27] proved that a smooth real algebraic curve of genus $g$ has at most $g+1$ topological components, and he constructed real algebraic curves of genus $g$ with $g+1$ components. In particular, a plane curve of degree $d$ has genus $g=(d-1)(d-2) / 2$ and there are real plane curves of degree $d$ with $g+1$ components. (An example is provided by Hilbert's quartic, which has genus 3.) Finer topological questions than enumerating the

\footnotetext{
${ }^{\dagger}$ With Powers, Reznick, and Scheiderer, we have shown that there are indeed 15 such ways.
} 


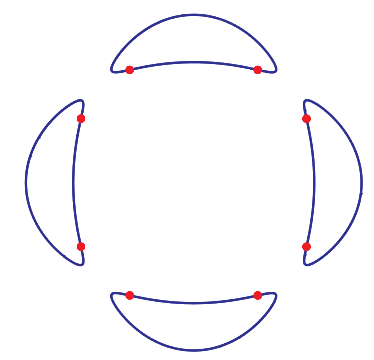

FiguRE 3. Hilbert's quartic: a plane quartic with 8 flexes

components leads to (part of) Hilbert's 16th problem [31, which asks for the determination of the topological types of smooth projectively embedded real algebraic varieties.

A variant concerns rational plane curves of degree $d$. A general rational plane curve of degree $d$ has $3(d-2)$ flexes and $g=(d-1)(d-2) / 2$ nodes. Theorem 4.2 implies that there exist real rational plane curves of degree $d$ with all $3(d-2)$ flexes real, which we call maximally inflected curves. See Section 5.1 for the connection. Such curves have at most $g-d+2$ of their nodes real, and there exist curves with the extreme values of 0 and of $g-d+2$ real nodes 39 . For example, a rational quartic $(d=4)$ has 6 flexes and $g=3$ nodes. If all 6 flexes are real, then at most one node is real. Figure 4 shows maximally inflected quartics with and 0 and 1 nodes. The flexes are marked by dots.
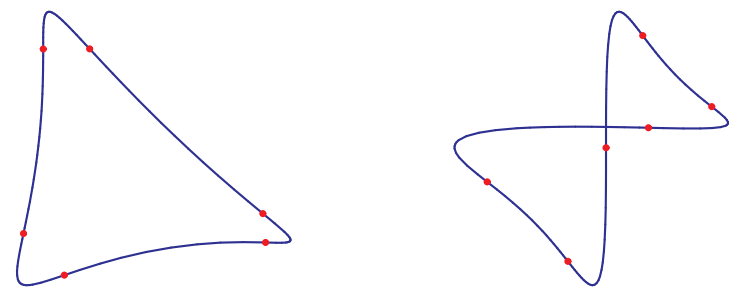

FigurE 4. Rational quartics with 6 real flexes

Recently, Huisman asked and answered a new question about real curves. A component $X$ of a real algebraic curve is a psuedoline if its homology class $[X]$ in $H^{1}\left(\mathbb{P}_{\mathbb{R}}^{n}, \mathbb{Z} / 2 \mathbb{Z}\right)$ is non-zero, and an oval otherwise.

QUESTION 3.2. Given a smooth (irreducible over $\mathbb{C}$ ) real algebraic curve $C$ in $\mathbb{P}^{n}$ of genus $g$ and degree $c$, how many real hypersurfaces of degree $d$ are tangent to at least $s$ components of $C$ with order of tangency at least $m$ ?

Let $\nu$ be that number, when there are finitely many such hypersurfaces.

Theorem 3.3 (Huisman [35, Theorem 3.1]). When $s=g$ and $g m=c d$, and the restriction

$$
H^{0}\left(\mathbb{P}^{n}, \mathcal{O}(d)\right) \longrightarrow H^{0}(C, \mathcal{O}(d))
$$

is an isomorphism, then $\nu$ is finite. Moreover, $\nu$ is non-zero if and only if

1. Both $m$ and $d$ are odd and $C$ consists of exactly $g$ psuedolines, or

2. $m$ is even and either $d$ is even or all components of $C$ are ovals. 
In case (1), $\nu=m^{g}$, and in case (2),

$$
\nu= \begin{cases}(g+1) m^{g} & \text { if } C \text { has } g+1 \text { components } \\ m^{g} & \text { if } C \text { has } g \text { components. }\end{cases}
$$

It is notable that this problem can only be stated over the real numbers.

3.2. The Stewart-Gough platform. The position of a rigid body in $\mathbb{R}^{3}$ has 6 degrees of freedom. This is exploited in robotics, giving rise to the Stewart-Gough platform [25, 80]. Specifically, suppose we have 6 fixed points $A_{1}, A_{2}, \ldots, A_{6}$ in space and 6 points $B_{1}, B_{2}, \ldots, B_{6}$ on a rigid body $B$ (the framework of Figure 5). The body is

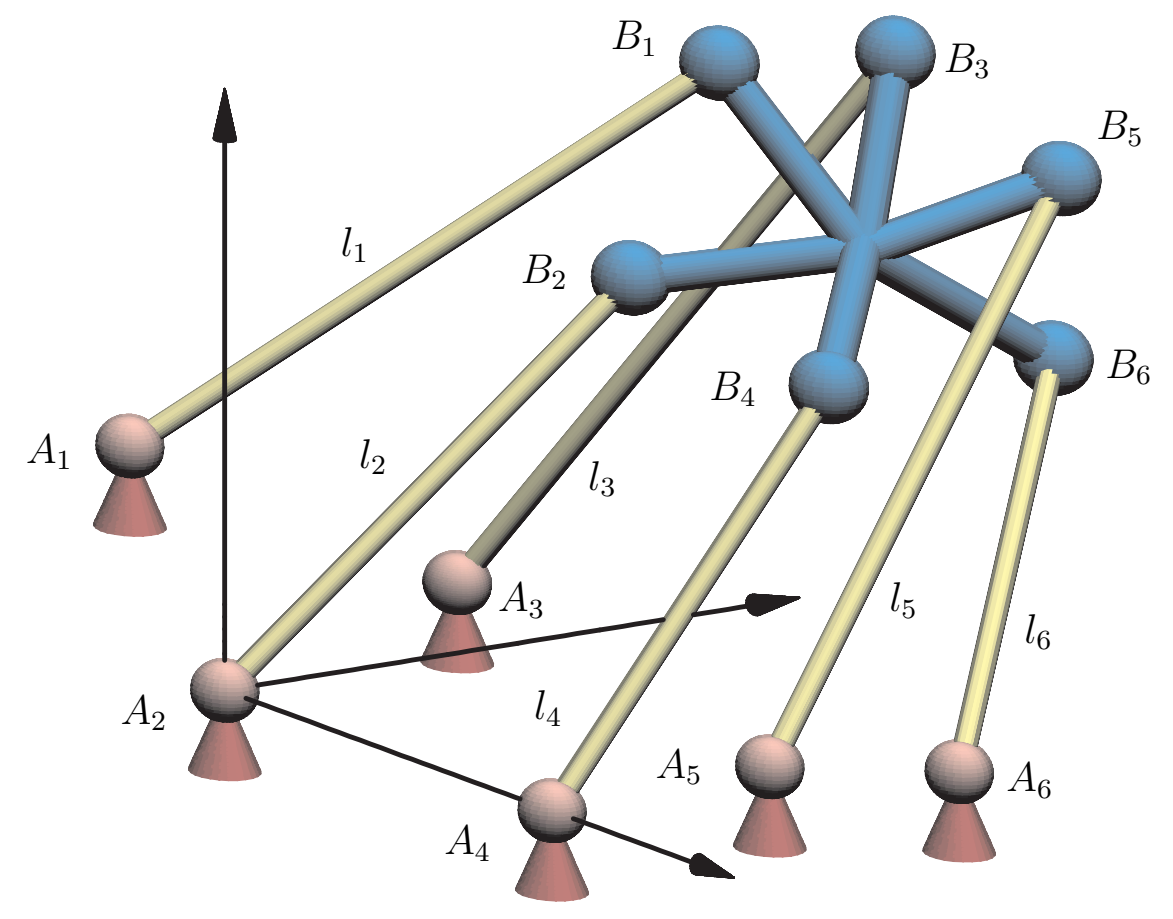

Figure 5. A Stewart Platform

controlled by varying each distance $l_{i}$ between the fixed point $A_{i}$ and the point $B_{i}$ on $B$. This may be accomplished by attaching rigid actuators to spherical joints located at the points $B_{i}$, or by suspending the platform from cables.

Given a position of the body $B$ in space, the distances $l_{1}, l_{2}, \ldots, l_{6}$ are uniquely determined. A fundamental problem is the inverse question: Given a platform (positions of the $A_{i}$ fixed and the relative positions of the $B_{i}$ specified) and a sextuple of distances $l_{1}, l_{2}, \ldots, l_{6}$, what is the position of the platform in space?

It had long been understood that several positions were possible for a given sextuple of lengths. An early work in 1897 showed there could be as many as 16 different positions [4. This leads to the following enumerative problem.

QUESTION 3.4. For a given Stewart platform, how many (complex) positions are there for a generic choice of the distances $l_{1}, l_{2}, \ldots, l_{6}$ ? How many of these can be real?

In the early 1990's, several approaches (numerical experimentation [59], intersection theory [62], Gröbner bases [48], resultants [53], and algebra [54]) each showed that there 
are 40 complex positions of a general Stewart platform. The obviously practical question of how many positions could be real remained open until 1998, when Dietmaier introduced a novel method to find a value of the distances $l_{1}, l_{2}, \ldots, l_{6}$ with all 40 positions real.

Theorem 3.5 (Dietmaier [14]). All 40 positions can be real.

Dietmaier's method will find future applications to other problems of this kind. He began with a formulation of the problem as a system of equations depending upon the distances $l_{1}, l_{2}, \ldots, l_{6}$. An initial solution for a given instance of the distances gave 6 real solutions and 17 pairs of complex conjugate solutions. He then used an ingenious algorithm to vary the distances in search of a configuration with all 40 solutions real.

This algorithm systematically varies the distances with the intention of increasing the number of real solutions. It proceeds in two stages. In the first stage, a pair of complex conjugate solutions $(x, \bar{x})$ are driven together, eventually creating a double solution, while at the same time the existing real solutions are kept bounded away from one another. At the formation of a double (necessarily real) solution, the distances are further incremented to create two new nearby real solutions $\left(x_{1}, x_{2}\right)$, which are then driven apart in the second stage. This procedure is repeated again with another pair of complex conjugate solutions, and et cetera. Figure 6 ilustrates the two stages.

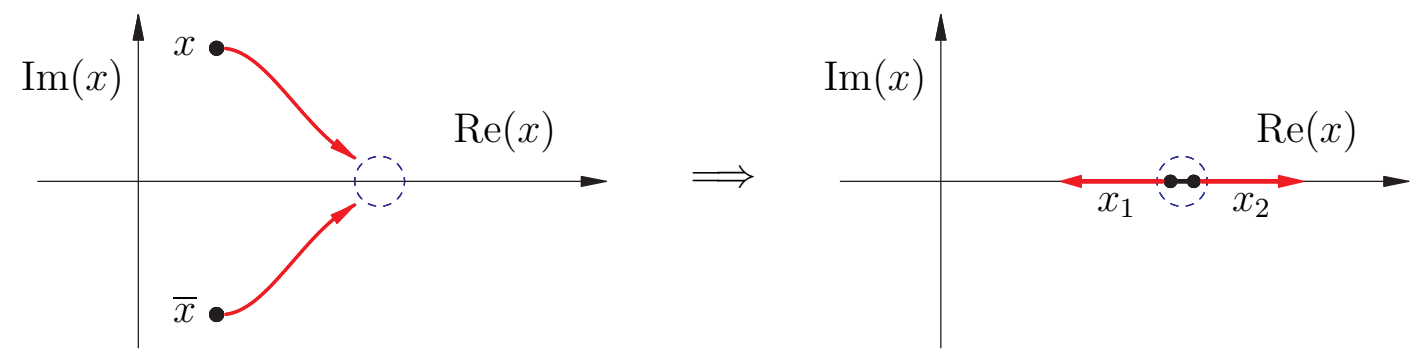

Figure 6. The two stages of Dietmaier's algorithm.

In each stage, Dietmaier accomplishes the given task (eg. colliding conjugate solutions) by linearizing the system at the current solutions and then solving a linear program for the optimal increment of the distances for the given goal. Changing the distances, he uses Newton's method beginning with the current solutions to find soutions for the new set of distances, and then repeats this procedure until the goal is acheived (eg. the conjugate pair collides). This is an application of numerical homotopy continuation [84].

While there is no guarantee that this method will even successfully collide two conjugate solutions, Dietmaier uses it to find a sextuple of distances with all 40 solutions real. While at each step the solutions are only numerical approximations to the actual solutions, the condition number $N$ guarantees the existence of a genuine solution within $1 / N$ of each approximate solution. Since the approximate real solutions were separated by more than $2 / N$, the requirement that non-real solutions occur in complex conjugate pairs forced these genuine solutions to be real.

3.3. Real rational cubics through 8 points in $\mathbb{P}_{\mathbb{R}}^{2}$. There are 12 singular (rational) cubic curves containing 8 general points in the plane. 
Theorem 3.6 (Degtyarev and Kharlamov [12, Proposition 4.7.3]). Given 8 general points in $\mathbb{P}_{\mathbb{R}}^{2}$, there are at least 8 singular real cubics containing them, and there are choices of the 8 points for which all 12 singular cubics are real.

Proof. Since a cubic equation in the plane has 10 coefficients, the space of cubics is identified with $\mathbb{P}^{9}$. The condition for a plane cubic to contain a given point is linear in these coefficients. Given 8 general points, these linear equations are independent and so there is a pencil $\left(\mathbb{P}^{1}\right)$ of cubics containing 8 general points in $\mathbb{P}^{2}$.

Two cubics $P$ and $Q$ in this pencil meet transversally in 9 points. Since curves in the pencil are given by $a P+b Q$ for $[a, b] \in \mathbb{P}^{1}$, any two curves in the pencil meet transversally in these 9 points. Let $Z$ be $\mathbb{P}^{2}$ blown up at these same 9 points. We have a map

$$
f: Z \longrightarrow \mathbb{P}^{1}
$$

where $f^{-1}([a, b])$ is the cubic curve defined by the polynomial $a P+b Q$.

Consider the Euler characteristic $\chi(Z)$ of $Z$ first over $\mathbb{C}$ and then over $\mathbb{R}$. Blowing up a smooth point on a surface replaces it with a $\mathbb{P}_{\mathbb{C}}^{1}$ and thus increases the Euler characteristic by 1 . Since $\chi\left(\mathbb{P}_{\mathbb{C}}^{2}\right)=3$, we see that $\chi(Z)=12$. The general fibre of $f$ is a smooth plane cubic which is homeomorphic to $S^{1} \times S^{1}$, and so has Euler characteristric 0. Thus only the singular fibres of $f$ contribute to the Euler characteristic of $Z$. Assume that the 8 points are in general position so there are only nodal cubics in the pencil. A nodal cubic has Euler characteristic 1. Thus there are 12 singular fibres of $f$ and hence 12 singular cubics meeting 8 general points in $\mathbb{P}_{\mathbb{C}}^{2}$.

Consider now the Euler characteristic of $Z_{\mathbb{R}}$. Blowing up a smooth point on a real surface replaces the point by $\mathbb{P}_{\mathbb{R}}^{1}=S^{1}$, and hence decreases the Euler characterstic by 1 . Since $\chi\left(\mathbb{P}_{\mathbb{R}}^{2}\right)=1$, we have $\chi\left(Z_{\mathbb{R}}\right)=1-9=-8$. A nonsingular real cubic is homeomorphic to either one or two disjoint copies of $S^{1}$, and hence has Euler characteristic 0. Again the Euler characteristic of $Z_{\mathbb{R}}$ is carried by its singular fibres. There are two nodal real cubics; either the node has two real branches or two complex conjugate branches so that the singular point is isolated. Call these curves real nodal and complex nodal, respectively. They are displayed in Figure 7. The real nodal curve is homoemorphic to a figure 8 and
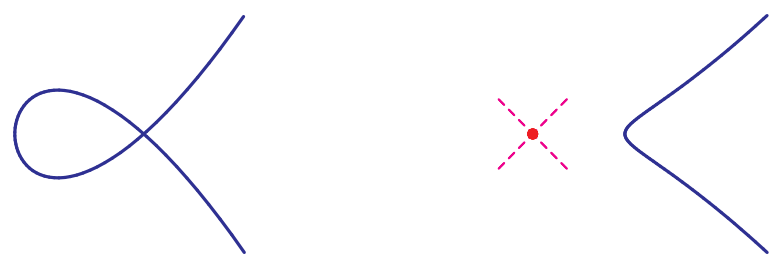

FiguRE 7. A real nodal and complex nodal curve

has Euler characteristic -1, while the complex nodal curve is the union of a $S^{1}$ with a point and so has Euler characteristic 1.

Among the singular fibres, we have

$$
\begin{aligned}
-8 & =\text { \#complex nodal }- \text { \#real nodal, with } \\
12 & \geq \text { \#complex nodal }+ \text { \#real nodal. }
\end{aligned}
$$


Thus there are at least 8 real nodal curves containing 8 general points in $\mathbb{P}_{\mathbb{R}}^{2}$. The pencil of cubics containing the 2 complex nodal cubics of Figure 8 has 10 real nodal cubics. Thus there are 12 real rational cubics containing any 8 of the 12 points in Figure 8 .

$$
\begin{aligned}
& \frac{(y+9 x-28)^{2}}{4}=(x-1) x^{2} \\
& \frac{(x+10 y-28)^{2}}{4}=y(y+1)^{2}
\end{aligned}
$$

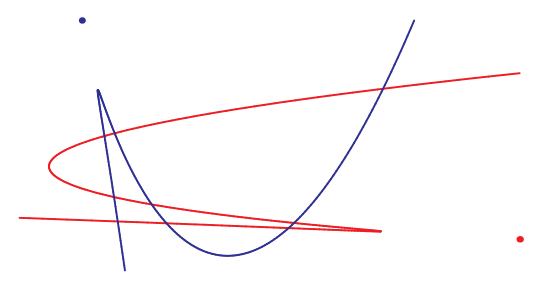

Figure 8. Complex nodal curves meeting in 9 points

REMARK 3.7. This classical problem of 12 plane cubics containing 8 points generalizes to the problem of enumerating rational plane curves of degree $d$ containing $3 d-1$ points. Let $N_{d}$ be the number of such curves, which satisfies the recursion [22]

$$
N_{d}=\sum_{d_{1}+d_{2}=d} N_{d_{1}} N_{d_{2}}\left[d_{1}^{2} d_{2}^{2}\left(\begin{array}{c}
3 d-4 \\
3 d_{1}-2
\end{array}\right)-d_{1}^{3} d_{2}\left(\begin{array}{c}
3 d-4 \\
3 d_{1}-1
\end{array}\right)\right] \text {. }
$$

The values $N_{1}=N_{2}=1$ are trivially fully real, and we have just seen that $N_{3}=12$ is fully real. The next case of $N_{4}=620$ (computed by Zeuthen [88]) seems quite challenging.

REMARK 3.8. The most interesting feature of Theorem 3.6 is the existence of a lower bound on the number of real solutions, which is a new phenomenon. In Section 6 we shall see evidence that this may be a pervasive feature of this field.

3.4. Common tangent lines to $2 n-2$ spheres in $\mathbb{R}^{n}$. Consider the following.

QUESTION 3.9. How many common tangent lines are there to $2 n-2$ spheres in $\mathbb{R}^{n}$ ?

For example, when $n=3$, how many common tangent lines are there to four spheres in $\mathbb{R}^{3}$ ? Despite its simplicity, this question does not seem to have been asked classically, but rather arose in discrete and computational geometry ${ }^{\dagger}$. The case $n=3$ was solved by Macdonald, Pach, and Theobald [51] and the general case more recently ${ }^{\S}$ [79].

THEOREM 3.10. $2 n-2$ general spheres in $\mathbb{R}^{n}(n \geq 3)$ have $3 \cdot 2^{n-1}$ complex common tangent lines, and there are $2 n-2$ such spheres with all common tangent lines real.

Figure 9 displays a configuration of 4 spheres in $\mathbb{R}^{3}$ with 12 real common tangents. The number $2 n-2$ is the dimension of the space of lines in $\mathbb{R}^{n}$ and is necessary for there to be finitely many common tangents.

Represent a line in $\mathbb{R}^{n}$ by a point $p$ on the line and its direction vector $v \in \mathbb{P}^{n-1}$. Imposing the condition

$$
p \cdot v=0
$$

${ }^{\dagger}$ The question of the maximal number of (real) common tangents to 4 balls was first formulated by David Larman 47] at DIMACS in 1990.

$\S$ This was solved during the DIMACS Workshop on Algorithmic and Quantitative Aspects of Real Algebraic Geometry in Mathematics and Computer Science. 


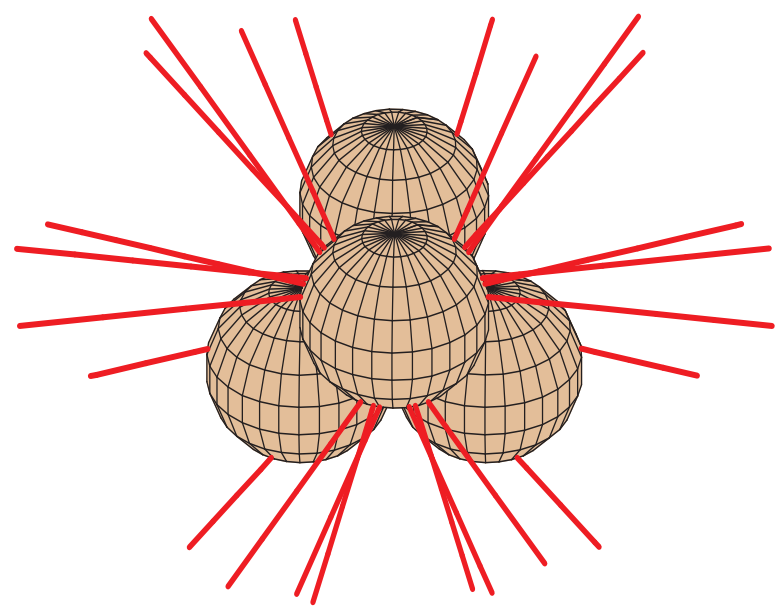

Figure 9. Four spheres with 12 common tangents

makes this representation unique. Here $\cdot$ is the usual Euclidean dot product. Write $v^{2}$ for $v \cdot v$. A line $(p, v)$ is tangent to the sphere with center $c$ and radius $r$ when

$$
v^{2} p^{2}-2 v^{2} p \cdot c+v^{2} c^{2}-(v \cdot c)^{2}-r^{2} v^{2}=0 .
$$

Suppose we have $2 n-2$ spheres with centers $c_{1}, c_{2}, \ldots, c_{2 n-2}$ and corresponding radii $r_{1}, r_{2}, \ldots, r_{2 n-2}$. Without any loss of generality, we may assume that the last sphere is centered at the origin and has radius $r$. Then its equation is

$$
v^{2} p^{2}-v^{2} r^{2}=0 \text {. }
$$

Subtracting this from the equations for the other spheres, we obtain the equations

$$
2 v^{2} p \cdot c_{i}=v^{2} c_{i}^{2}-\left(v \cdot c_{i}\right)^{2}-v^{2}\left(r_{i}^{2}-r^{2}\right)
$$

for $i=1,2, \ldots, 2 n-3$.

These last equations are linear in $p$. If the centers $c_{1}, c_{2}, \ldots, c_{n}$ are linearly independent (which they are, by our assumption on generality), then we use the corresponding equations to solve for $v^{2} p$ as a homogeneous quadratic in $v$. Substituting this into the equations (3.1) and (3.2) gives a cubic and a quartic in $v$, and substituting the expression for $v^{2} p$ into (3.3) for $i=n+1, n+2, \ldots, 2 n-3$ gives $n-3$ quadratics in $v$. By Bézout's Theorem, if there are finitely many complex solutions to these equations, their number is bounded by $3 \cdot 4 \cdot 2^{n-3}=3 \cdot 2^{n-1}$.

This upper bound is attained with all solutions real. Suppose that the spheres all have the same radius, $r$, and the first four have centers

$$
\begin{aligned}
& c_{1}:=(1,1,1,0, \ldots, 0), \\
& c_{2}:=(1,-1,-1,0, \ldots, 0), \\
& c_{3}:=(-1,1,-1,0, \ldots, 0), \\
& c_{4}:=(-1,-1,1,0, \ldots, 0),
\end{aligned}
$$

and subsequent centers are at the points $\pm a e_{j}$ for $j=4,5, \ldots, n$, where $e_{1}, e_{2}, \ldots, e_{n}$ is the standard basis for $\mathbb{R}^{n}$. Let $\gamma:=a^{2}(n-1) /\left(a^{2}+n-3\right)$, which is positive. 
Theorem 3.11 ([79, Theorem 5]). When

$$
\operatorname{ar}\left(r^{2}-3\right)(3-\gamma)\left(a^{2}-2\right)\left(r^{2}-\gamma\right)\left((3-\gamma)^{2}+4 \gamma-4 r^{2}\right) \neq 0,
$$

there are exactly $3 \cdot 2^{n-1}$ complex lines tangent to the spheres. If we have

(a) $\frac{1}{4}(3-\gamma)^{2}+\gamma>r^{2}>\gamma$ and

(b) $\frac{n-1}{n-4}+2>a^{2}>2$,

then all the $3 \cdot 2^{n-1}$ lines are in fact real.

Theorem 3.10 is false when $n=2$. There are 4 lines tangent to 2 circles in the plane, and all may be real. The argument given for Theorem 3.10 fails because the centers of the spheres do not affinely span $\mathbb{R}^{2}$. This case of $n=2$ does generalize, though.

THEOREM 3.12 (Megyesi [52]). Four unit spheres in $\mathbb{R}^{3}$ whose centers are coplanar but otherwise general have 12 common complex tangents. At most 8 of these 12 are real.

REMARK 3.13. This problem of common tangents to 4 spheres with equal radii and coplanar centers gives an example of an enumerative geometric problem that is not fully real. We do not feel this contradicts the observation that there are no enumerative problems not known to be fully real, as the spheres are not sufficiently general.

\section{Schubert Calculus}

The classical Schubert calculus of enumerative geometry is concerned with questions of enumerating linear subspaces of a vector space or projective space that satisfy incidence conditions imposed by other linear subspaces. A non-trivial instance is the question posed at the beginning of Section 3.

Question 4.1. How many lines in space meet four general lines $\ell_{1}, \ell_{2}, \ell_{3}$, and $\ell_{4}$ ?

Three pairwise skew lines $\ell_{1}, \ell_{2}$, and $\ell_{3}$ lie on a unique smooth quadric surface $Q$. There are two families of lines that foliate $Q$ - one family includes $\ell_{1}, \ell_{2}$, and $\ell_{3}$ and the other consists of the lines meeting each of $\ell_{1}, \ell_{2}$, and $\ell_{3}$. The fourth line $\ell_{4}$ meets $Q$ in two points, and each of these points determines a line in the second family. Thus there are 2 lines $\mu_{1}, \mu_{2}$ in space that meet general lines $\ell_{1}, \ell_{2}, \ell_{3}$, and $\ell_{4}$. Figure 10 shows this configuration. Figure 10 also shows how the two lines can be real-if $\ell_{4}$ meets $Q$ in two real points. (The two lines are complex when $\ell_{4}$ meets $Q$ in two complex conjugate points.)

The classical Schubert calculus is a vast generalization of this problem of four lines. In the 1980's Speiser suggested to Fulton that the classical Schubert calculus may be a good testing ground for Question 3.1. This was also considered by Chiavacci and EscamillaCastillo 8]. We will discuss increasingly more general versions of the Schubert calculus, and the status of Question 3.1 for each.

Consider first more general problems involving lines. The space of lines in $\mathbb{P}^{n}$ is a smooth projective variety of dimension $2 n-2$ called the Grassmannian of lines in $\mathbb{P}^{n}$. The set of lines meeting a linear subspace $L$ of dimension $n-1-l$ has codimension $l$ in the Grassmannian. Thus given general linear subspaces $L_{1}, L_{2}, \ldots, L_{s}$ of $\mathbb{P}^{n}$ with $\operatorname{dim} L_{i}=n-1-l_{i}$ where $l_{1}+l_{2}+\cdots+l_{s}=2 n-2$, we expect (and there are indeed) finitely

many lines in $\mathbb{P}^{n}$ meeting each linear subspace $L_{1}, L_{2}, \ldots, L_{s}$. Schubert 65] discovered 


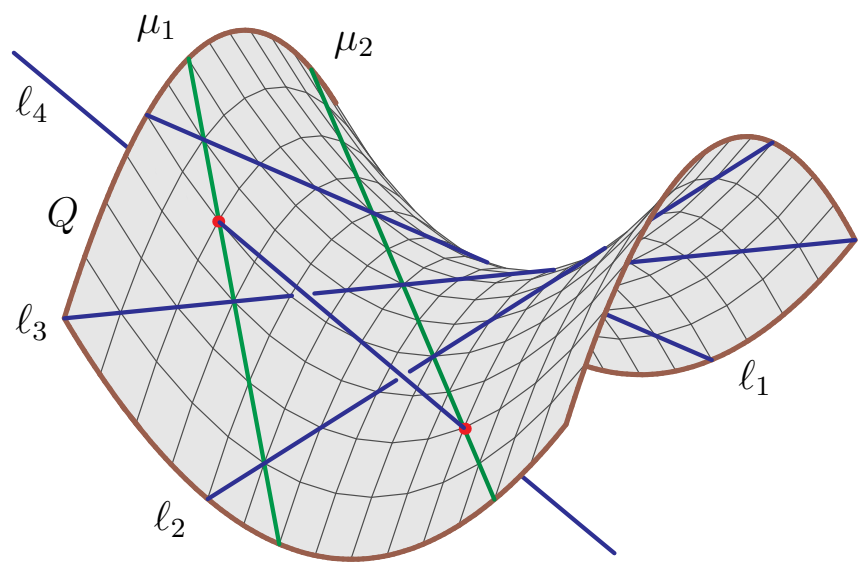

Figure 10. The two lines meeting four general lines in space.

algorithms for computing this number $d\left(l_{1}, l_{2}, \ldots, l_{s}\right)$ of lines. For example, if each $l_{i}=1$, so that $s=2 n-2$, then this number is the $n$th Catalan number ${ }^{\dagger}$

$$
C_{n}=\frac{1}{n}\left(\begin{array}{c}
2 n-2 \\
n-1
\end{array}\right) \text {. }
$$

Enumerative problems of lines in $\mathbb{P}^{n}$ meeting general linear subspaces furnished the first infinite family of non-trivial enumerative problems known to be fully real.

TheOREM $4.2\left(\left[\mathbf{6 9}\right.\right.$, Theorem C]). Given positive integers $l_{1}, l_{2}, \ldots, l_{s}$ with $l_{1}+l_{2}+$ $\cdots+l_{s}=2 n-2$, there exist linear subspaces $L_{1}, L_{2}, \ldots, L_{s}$ of $\mathbb{R P}^{n}$ with $\operatorname{dim} L_{i}=n+1-l_{i}$ such that there are exactly $d\left(l_{1}, l_{2}, \ldots, l_{s}\right)$ complex lines meeting each subspace $L_{i}$, and each of these lines are real.

4.1. The special Schubert calculus. More generally, we may ask how many linear subspaces of a fixed dimension meet general linear subspaces. We formulate this question in terms of linear subspaces of a vector space.

The set of $k$-dimensional subspaces ( $k$-planes) of an $n$-dimensional vector space forms the Grassmannian of $k$-planes in $n$-space, $\operatorname{Gr}(k, n)$, a smooth projective variety of dimension $k(n-k)$. Those $k$-planes meeting a linear subspace $L$ of dimension $n-k+1-l$ non-trivially (that is, the intersection has positive dimension) form the special Schubert subvariety $\Omega(L)$ of $\operatorname{Gr}(k, n)$ which has codimension $l$. The special Schubert calculus is concerned with the following question.

Question 4.3. Given general linear subspaces $L_{1}, L_{2}, \ldots, L_{s}$ of $\mathbb{C}^{n}$ with $\operatorname{dim} L_{i}=$ $n-k+1-l_{i}$ where $l_{1}+l_{2}+\cdots+l_{s}=k(n-k)$, how many $k$-planes $K$ meet each subspace $L_{i}$ non-trivially, that is, satisfy

$$
K \cap L_{i} \neq\{0\} \quad i=1,2, \ldots, s ?
$$

The condition (4.2) is expressed in the global geometry of $\operatorname{Gr}(k, n)$ as the number of points in the intersection of the special Schubert varieties

$$
\Omega\left(L_{1}\right) \cap \Omega\left(L_{2}\right) \cap \cdots \cap \Omega\left(L_{s}\right),
$$

${ }^{\dagger}$ This indexing of the Catalan numbers is shifted from that of some other authors. 
when the intersection is transverse. (A general theorem of Kleiman 42 guarantees transversality when the $L_{i}$ are in general position, and also implies transversality for the other intersections considered in this section.)

There are algorithms due to Schubert 66] (when each $l_{i}=1$ ) and Pieri [57] to compute the expected number of solutions. When each $l_{i}=1$, Schubert 64 showed that the number of solutions is equal to

$$
d(n, k):=\frac{1 ! 2 ! \cdots(k-1) ! \cdot[k(n-k)] !}{(n-k) !(n-k+1) ! \cdots(n-1) !} .
$$

A line in $\mathbb{P}^{n}$ is a 2-plane in $(n+1)$-space and two linear subspaces in $\mathbb{P}^{n}$ meet if and only if the corresponding linear subspaces in $(n+1)$-space have a non-trivial intersection. Thus the problem of lines in projective space corresponds to the case $k=2$ of the special Schubert calculus. While the geometric problem generalizes easily from $k=2$ to arbitrary values of $k$, the proof of Theorem 4.2 does not. There is, however, a relatively simple argument that this special Schubert calculus is fully real.

Theorem 4.4 ([72, Theorem 1]). Suppose $n>k>0$ and $l_{1}, l_{2}, \ldots, l_{s}$ are positive integers with $l_{1}+l_{2}+\cdots+l_{s}=k(n-k)$. Then there are linear subspaces $L_{1}, L_{2}, \ldots, L_{s}$ of $\mathbb{R}^{n}$ in general position with $\operatorname{dim} L_{i}=n-k+1-l_{i}$ such that each of the a priori complex $k$-planes $K$ satisfying (4.2) are in fact real.

We present an elementary proof of this result in the important special case when each $l_{i}=1$ so that the conditions are simple, meaning each $\Omega\left(L_{i}\right)$ has codimension 1 . This proof generalizes to show that some other classes of enumerative problems in the Schubert calculus are fully real (see Sections 4.2.2 and 4.2.3). This generalization constructs sufficiently many real solutions using a limiting argument, as in Section 2.2. Just as the arguments of Section 2.2 were linked to the homotopy algorithms of Huber and Sturmfels, the proof of Theorem 4.4 leads to numerical homotopy methods for solving these problems 32, 34].

We develop further geometric properties of Grassmann varieties. The $k$ th exterior power of the embedding $K \rightarrow \mathbb{C}^{n}$ of a $k$-plane $K$ into $\mathbb{C}^{n}$ gives the embedding

$$
\mathbb{C} \simeq \wedge^{k} K \longrightarrow \wedge^{k} \mathbb{C}^{n}
$$

whose image is a line in $\wedge^{k} \mathbb{C}^{n}$ and thus a point in the projective space $\mathbb{P}\left(\wedge^{k} \mathbb{C}^{n}\right) \simeq \mathbb{P}\left(\begin{array}{l}n \\ k\end{array}\right)-1$. This point determines the $k$-plane $K$ uniquely. The Plücker embedding is the resulting projective embedding of the Grassmannian

$$
\operatorname{Gr}(k, n) \longrightarrow \mathbb{P}^{\left(\begin{array}{l}
n \\
k
\end{array}\right)-1} .
$$

The $\left(\begin{array}{l}n \\ k\end{array}\right)$ homogeneous Plücker coordinates for the Grassmannian in this embedding are realized concretely as follows. Represent a $k$-plane $K$ as the row space of a $k \times n$ matrix, also written $K$. A maximal minor of $K=\left(a_{i j}\right)$ is the determinant of a $k \times k$ submatrix of $K$ : Given a choice of columns $\alpha$ : $1 \leq \alpha_{1}<\alpha_{2}<\cdots<\alpha_{k} \leq n$, set

$$
p_{\alpha}(K):=\operatorname{det}\left[\begin{array}{cccc}
a_{1, \alpha_{1}} & a_{1, \alpha_{2}} & \cdots & a_{1, \alpha_{k}} \\
a_{2, \alpha_{1}} & a_{2, \alpha_{2}} & \cdots & a_{2, \alpha_{k}} \\
\vdots & \vdots & \ddots & \vdots \\
a_{k, \alpha_{1}} & a_{k, \alpha_{2}} & \cdots & a_{k, \alpha_{k}}
\end{array}\right] \text {. }
$$


The vector $\left(p_{\alpha}(K)\right)$ of $\left(\begin{array}{l}n \\ k\end{array}\right)$ maximal minors of $K$ defines the map (4.4) giving Plücker coordinates for $K$. Let $\left(\begin{array}{c}{[n]} \\ k\end{array}\right)$ be the collection of these indices of Plücker coordinates.

The indices $\left(\begin{array}{c}{[n]} \\ k\end{array}\right)$ have a natural Bruhat order

$$
\beta \leq \alpha \Longleftrightarrow \beta_{j} \leq \alpha_{j} \text { for } j=1,2, \ldots, k .
$$

The Schubert variety $\Omega_{\alpha}$ is

$$
\Omega_{\alpha}=\left\{K \in \operatorname{Gr}(k, n) \mid p_{\beta}(K)=0 \text { for } \beta \not L \alpha\right\} .
$$

This has dimension $|\alpha|:=\sum_{j}\left(\alpha_{j}-j\right)$.

The relevance of the Plücker embedding to Question 4.3 when $l_{i}=1$ is seen as follows. Let $L$ be a $(n-k)$-plane, represented as the row space of a $(n-k)$ by $n$ matrix, also written $L$. Then a general $k$-plane $K$ meets $L$ non-trivially if and only if

$$
\operatorname{det}\left[\begin{array}{c}
K \\
L
\end{array}\right]=0 \text {. }
$$

Laplace expansion along the rows of $K$ gives

$$
0=\operatorname{det}\left[\begin{array}{c}
K \\
L
\end{array}\right]=\sum_{\alpha \in\left(\begin{array}{c}
{[n]} \\
k
\end{array}\right)} p_{\alpha}(K) L_{\alpha},
$$

where $L_{\alpha}$ is the appropriately signed minor of $L$ given by the columns complementary to $\alpha$. Hence the set $\Omega(L)$ of $k$-planes that meet the $(n-k)$-plane $L$ non-trivially is a hyperplane section of the Grassmannian in its Plücker embedding.

Thus the set of $k$-planes meeting $k(n-k)$ general $(n-k)$-planes non-trivially is a complementary linear section of the Grassmannian, and so the number $d(k, n)$ of such $k$-planes is the degree of the Grassmannian in its Plücker embedding. More generally, if $\alpha \in\left(\begin{array}{c}{[n]} \\ k\end{array}\right)$ and $L_{1}, L_{2}, \ldots, L_{|\alpha|}$ are general $(n-k)$-planes, then the number of points in the intersection

$$
\Omega_{\alpha} \cap \Omega\left(L_{1}\right) \cap \Omega\left(L_{2}\right) \cap \cdots \cap \Omega\left(L_{|\alpha|}\right)
$$

is the degree $d(\alpha)$ of the Schubert variety $\Omega_{\alpha}$, which we now compute.

An intersection $X \cap Y$ is generically transverse if $X$ and $Y$ meet transversally along an open subset of every component of $X \cap Y$. When $\beta, \alpha \in\left(\begin{array}{c}{[n]} \\ k\end{array}\right)$ satisfy $\beta<\alpha$ but there is no index $\gamma$ with $\beta<\gamma<\alpha$, then we write $\beta \lessdot \alpha$. The following fact is elementary and due to Schubert.

THEOREM 4.5. Let $\alpha \in\left(\begin{array}{c}{[n]} \\ k\end{array}\right)$ and set $H_{\alpha}$ to be the hyperplane defined by $p_{\alpha}=0$. Then

$$
\Omega_{\alpha} \cap H_{\alpha}=\bigcup_{\beta \lessdot \alpha} \Omega_{\beta}
$$

and the intersection is generically transverse.

In fact this intersection is transverse along $\Omega_{\beta}^{\circ}:=\Omega_{\beta}-\bigcup_{\delta<\beta} \Omega_{\delta}$. We obtain the recursion for the degree $d(\alpha)$ of the Schubert variety $\Omega_{\alpha}$

$$
d(\alpha)=\sum_{\beta \lessdot \alpha} d(\beta) .
$$


Since the minimal Schubert variety is a point (which has degree 1), this gives a conceptual formula for $d(\alpha)$. Let $\hat{0}=(1,2, \ldots, k)$ be the minimal element in the Bruhat order.

$$
d(\alpha)=\text { the number of saturated chains in the Bruhat order from } \hat{0} \text { to } \alpha \text {. }
$$

Figure 11 displays both the Bruhat order for $k=3$ and $n=6$ (on the left) and the degrees of the corresponding Schubert varieties (on the right).
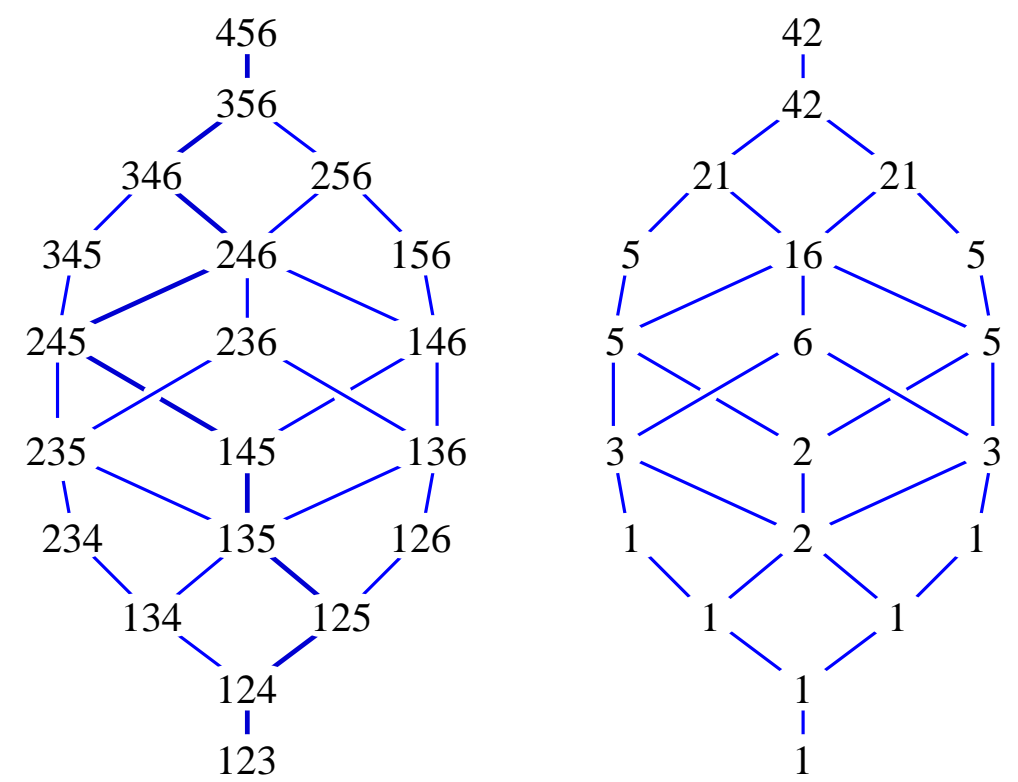

FiguRE 11. Bruhat order and degrees of Schubert varieties, $k=3, n=6$

Consider the action of the non-zero real numbers $\mathbb{R}^{\times}$on $\mathbb{R}^{n}$

$$
t . e_{j}:=t^{j} \cdot e_{j}
$$

where $t \in \mathbb{R}^{\times}$and $e_{1}, e_{2}, \ldots, e_{n}$ is a basis for $\mathbb{R}^{n}$ (corresponding to the rows of the $n \times n$ identity matrix). Let $L$ be a $(n-k)$-plane. By (4.6), the equation for a $k$-plane $K$ to meet $t . L$ non-trivially is

$$
0=\sum_{\beta} t^{\left(\begin{array}{c}
n+1 \\
2
\end{array}\right)-|\beta|} L_{\beta} p_{\beta}(K) \text {. }
$$

For $K \in \Omega_{\alpha}$, the sum is over $\beta \leq \alpha$ by (4.5). Removing the common factor $t^{\left(\begin{array}{c}n+1 \\ 2\end{array}\right)-|\alpha|}$ gives

$$
0=\sum_{\beta \leq \alpha} t^{|\alpha|-|\beta|} L_{\beta} p_{\beta}(K) .
$$

The case $l_{i}=1$ of Theorem 4.4 is implied by the case $\alpha=(n-k+1, \ldots, n-1, n)=\hat{1}$ $\left(\Omega_{\hat{1}}=\operatorname{Gr}(k, n)\right)$ of the following theorem.

Theorem $4.6\left(\mathbb{7 6}\right.$, Theorem 4.2]). Let $L \subset \mathbb{R}^{n}$ be a $(n-k)$-plane, none of whose Plücker coordinates vanishes. Then there exist real numbers $t_{1}, t_{2}, \ldots, t_{k(n-k)} \in \mathbb{R}^{\times}$such that for every $\alpha \in\left(\begin{array}{c}{[n]} \\ k\end{array}\right)$ the intersection

$$
\Omega_{\alpha} \cap \Omega\left(t_{1} . L\right) \cap \Omega\left(t_{2} . L\right) \cap \cdots \cap \Omega\left(t_{|\alpha|} . L\right)
$$

is transverse (so it contains $d(\alpha)$ points) with all points real. 
ProOF. We induct on $m$ to construct numbers $t_{1}, t_{2}, \ldots, t_{k(n-k)} \in \mathbb{R}^{\times}$having the property that, for all $\alpha \in\left(\begin{array}{c}{[n]} \\ k\end{array}\right)$ with $|\alpha|=m$,

$$
\Omega_{\alpha} \cap \Omega\left(t_{1} . L\right) \cap \Omega\left(t_{2} . L\right) \cap \cdots \cap \Omega\left(t_{m} . L\right)
$$

is transverse (over $\mathbb{C}$ ) and each of its $d(\alpha)$ points are real.

The case $m=0$ is trivial, as $|\alpha|=0$ implies that $\alpha=\hat{0}$ and $\Omega_{\hat{0}}$ is a single (real) point in $\operatorname{Gr}(k, n)$. Suppose we have constructed $t_{1}, t_{2}, \ldots, t_{m} \in \mathbb{R}^{\times}$with the above properties.

Let $\alpha \in\left(\begin{array}{c}{[n]} \\ k\end{array}\right)$ with $|\alpha|=m+1$ and consider

$$
\left(\sum_{\beta \lessdot \alpha} \Omega_{\beta}\right) \cap\left(\Omega\left(t_{1} . L\right) \cap \cdots \cap \Omega\left(t_{m} . L\right)\right)=\sum_{\beta \lessdot \alpha} \Omega_{\beta} \cap \Omega\left(t_{1} . L\right) \cap \cdots \cap \Omega\left(t_{m} . L\right) .
$$

Each summand in the second sum is transverse (over $\mathbb{C}$ ) and consists of $d(\beta)$ real points. The intersection on the left will be transverse and consist of $d(\alpha)=\sum_{\beta \lessdot \alpha} d(\beta)$ real points only if no two summands in the second sum share a point in common.

If two summands $\beta, \gamma$ with $\beta \neq \gamma$ share a point, then

$$
\Omega_{\beta} \cap \Omega_{\gamma} \cap \Omega\left(t_{1} \cdot L\right) \cap \Omega\left(t_{2} \cdot L\right) \cap \cdots \cap \Omega\left(t_{m} . L\right)
$$

is non-empty. By (4.5), $\Omega_{\beta} \cap \Omega_{\gamma}$ is contained in a union of Schubert varieties of dimension less than $m$. By (4.10) the condition for a fixed $k$-plane $K \in \Omega_{\delta}$ to meet $t . L$ non-trivially is a polynomial of degree at most $|\delta|$ in $t$. Thus a fixed $k$-plane $K \in \Omega_{\delta}$ lies in at most $|\delta|$ distinct $(n-k)$-planes in the family t.L. Hence the intersection (4.12) is empty, and so the summands in the second sum of (4.11) are disjoint. This argument also shows that the points in the summand indexed by $\beta$ lie in $\Omega_{\beta}^{\circ}=\Omega_{\beta}-\bigcup_{\delta<\beta} \Omega_{\delta}$.

For $t \in \mathbb{R}$, let $Z_{t} \subset \Omega_{\alpha}$ be the set of $k$-planes $K$ satisfying the polynomial (4.10). For $t \neq 0$, we have $Z_{t}=\Omega_{\alpha} \cap \Omega(t . L)$. Since $L$ has no vanishing Plücker coordinates, the constant term of that polynomial is $p_{\alpha}(K)$, and so by Theorem 4.5,

$$
Z_{0}=\Omega_{\alpha} \cap H_{\alpha}=\sum_{\beta \lessdot \alpha} \Omega_{\beta}
$$

Thus $Z_{0}$ meets

$$
\Omega\left(t_{1} . L\right) \cap \Omega\left(t_{2} . L\right) \cap \cdots \cap \Omega\left(t_{m} . L\right)
$$

transversally (over $\mathbb{C}$ ) in $d(\alpha)$ real points. We see that there exists $\epsilon_{\alpha}>0$ such that if $0<t \leq \epsilon_{\alpha}$, then $Z_{t}$ meets (4.13) transversally (over $\mathbb{C}$ ) in $d(\alpha)$ real points. Since the intersection defining $Z_{0}$ is transverse along each $\Omega_{\beta}^{\circ}$ for $\beta \lessdot \alpha$, we may assume that for $0<t \leq \epsilon_{\alpha}$

$$
\Omega_{\alpha} \cap \Omega(t . L) \cap \Omega\left(t_{1} . L\right) \cap \cdots \cap \Omega\left(t_{m} . L\right)
$$

is transverse (over $\mathbb{C}$ ) and consists of $d(\alpha)$ real points.

Let $t_{m+1}=\min \left\{\epsilon_{\alpha}:|\alpha|=m+1\right\}$. Then for any $\alpha \in\left(\begin{array}{c}{[n]} \\ k\end{array}\right)$ with $|\alpha|=m+1$,

$$
\Omega_{\alpha} \cap \Omega\left(t_{1} . L\right) \cap \cdots \cap \Omega\left(t_{m} . L\right) \cap \Omega\left(t_{m+1} . L\right)
$$

is transverse (over $\mathbb{C}$ ) and consists of $d(\alpha)$ real points. 
4.2. Further extensions of the Schubert calculus. This special Schubert calculus admits further extensions, some of which are known to be fully real. Interestingly, some problems involving the Lagrangian Grassmannian 4.2.4 are known to be fully unreal, a new phenomenon. Further investigation is encouraged; in particular, the Lagrangian Schubert calculus may yield the first class of enumerative which are not fully real.

4.2.1. General Schubert calculus. A flag in $n$-space is a sequence of linear subspaces $F_{\bullet}: F_{1} \subset F_{2} \subset \cdots \subset F_{n}$ with $\operatorname{dim} F_{i}=i$. Given $\alpha \in\left(\begin{array}{c}{[n]} \\ k\end{array}\right)$, the Schubert condition of type $\alpha$ on a $k$-plane $K$ imposed by the flag $F_{\bullet}$ is

$$
\operatorname{dim} K \cap F_{n+1-\alpha_{j}} \geq k+1-j \quad \text { for } \quad j=1,2, \ldots, n .
$$

The Schubert variety $X_{\alpha} F_{\bullet} \subset \operatorname{Gr}(k, n)$ is the set of all $k$-planes $K$ satisfying (4.14).

We relate this to the definitions of Section 4.1. Let $e_{1}, e_{2}, \ldots, e_{n}$ be a basis for $\mathbb{R}^{n}$ and for its complexification $\mathbb{C}^{n}$. Defining $F_{i}$ to be the linear span of $e_{1}, e_{2}, \ldots, e_{i}$ gives the flag $F_{\bullet}$. Then the Schubert variety $X_{\alpha} F_{\bullet}$ is $\Omega_{\alpha^{\vee}}$, where $\alpha_{j}^{\vee}=n+1-\alpha_{k+1-j}$ for each $j$, and so the codimension of $X_{\alpha} F_{\bullet}$ is $|\alpha|$. A special Schubert condition is when $\alpha=(1, \ldots, k-1, k+l)$ so that $X_{\alpha} F_{\bullet}=\Omega\left(F_{n-k+1-l}\right)$.

The general problem of the classical Schubert calculus of enumerative geometry asks, given $\alpha^{1}, \alpha^{2}, \ldots, \alpha^{s} \in\left(\begin{array}{c}{[n]} \\ k\end{array}\right)$ with $\left|\alpha^{1}\right|+\left|\alpha^{2}\right|+\cdots+\left|\alpha^{s}\right|=k(n-k)$ and general flags $F_{\bullet}{ }^{1}, F_{\bullet}^{2}, \ldots, F_{\bullet}^{s} \subset \mathbb{C}^{n}$, how many points are there in the intersection ${ }^{\dagger}$

$$
X_{\alpha^{1}} F_{\bullet}^{1} \cap X_{\alpha^{2}} F_{\bullet}^{2} \cap \cdots \cap X_{\alpha^{s}} F_{\bullet}^{s} ?
$$

There are algorithms due to Pieri [57 and Giambelli [23 to compute these numbers. Other than the case when the $\alpha^{i}$ are indices of special Schubert varieties, it remains open whether the general Schubert calculus is fully real. (See [74] and [71] for some cases.)

4.2.2. Quantum Schubert calculus. The space $\mathcal{M}_{k, n}^{q}$ of degree $q$ maps $M: \mathbb{P}^{1} \rightarrow \operatorname{Gr}(k, n)$ is a smooth quasi-projective variety [9]. A point $t \in \mathbb{P}^{1}$ and a Schubert variety $X_{\alpha} F_{\bullet}$ together impose a quantum Schubert condition on maps $M \in \mathcal{M}_{k, n}^{q}$,

$$
M(t) \in X_{\alpha} F_{\bullet} .
$$

The set of such maps has codimension $|\alpha|$ in $\mathcal{M}_{k, n}^{q}$. The quantum Schubert calculus of enumerative geometry asks the following question.

Question 4.7. Given $\alpha^{1}, \alpha^{2}, \ldots, \alpha^{s} \in\left(\begin{array}{c}{[n]} \\ k\end{array}\right)$ with $\left|\alpha^{1}\right|+\left|\alpha^{2}\right|+\cdots+\left|\alpha^{s}\right|=\operatorname{dim} \mathcal{M}_{k, n}^{q}=$ $q n+k(n-k)$, how many maps $M \in \mathcal{M}_{k, n}^{q}$ satisfy

$$
M\left(t_{i}\right) \in X_{\alpha^{i}} F_{\bullet}^{i} \quad \text { for } i=1,2, \ldots, s,
$$

where $t_{1}, t_{2}, \ldots, t_{s} \in \mathbb{P}^{1}$ are general and $F_{\bullet}^{1}, F_{\bullet}^{2}, \ldots, F_{\bullet}^{s}$ are general flags?

Algorithms to compute this number were proposed by Vafa [83 and Intriligator [36] and proven by Siebert and Tian [67] and by Bertram [3]. A simple quantum Schubert condition defines a subvariety of codimension 1 ,

$$
M(t) \cap L \neq\{0\},
$$

where $L$ is a $(n-k)$-plane. Let $d(q ; k, n)$ be the number of maps $M \in \mathcal{M}_{k, n}^{q}$ satisfying $\operatorname{dim} \mathcal{M}_{k, n}^{q}$-many general simple quantum Schubert conditions (4.16). A combinatorial formula for this number was given by Ravi, Rosenthal, and Wang [60. For a survey of this particular enumerative problem and its importance to linear systems theory, see [77.

\footnotetext{
$\dagger$ In this survey flags are general when the corresponding intersection is transverse.
} 
TheOREM 4.8 ( $\left[73\right.$, Theorem 1.1]). Let $q \geq 0$ and $n>k>0$ and $\operatorname{set} N:=\operatorname{dim} \mathcal{M}_{k, n}$. Then there exist $t_{1}, t_{2}, \ldots, t_{N} \in \mathbb{R P}^{1}$ and $(n-k)$-planes $L_{1}, L_{2}, \ldots, L_{N} \subset \mathbb{R}^{n}$ such that there are exactly $d(q ; k, n)$ maps $M \in \mathcal{M}_{k, n}^{q}(\mathbb{C})$ satisfying

$$
M\left(t_{i}\right) \cap L_{i} \neq\{0\} \quad \text { for } i=1,2, \ldots, N
$$

and all of them are real.

As with the classical Schubert calculus, the question of whether the general quantum Schubert calculus is fully real remains open.

4.2.3. Schubert calculus of flags. Let $\mathbf{a}:=0<a_{1}<\cdots<a_{r}<a_{r+1}=n$ be a sequence of integers. The manifold of partial flags in $n$-space (or the flag manifold) $\mathbb{F} \ell_{\mathbf{a}}$ is the collection of partial flags of subspaces

$$
E_{\bullet}: E_{a_{1}} \subset E_{a_{2}} \subset \cdots \subset E_{a_{r}}
$$

in $n$-space, where $\operatorname{dim} E_{a_{i}}=a_{i}$. A complete flag $F_{\bullet}$ is when $\mathbf{a}=1,2, \ldots, n-1$.

The Schubert varieties of $\mathbb{F} \ell_{\mathbf{a}}$ are indexed by permutations $w \in \mathcal{S}_{n}$, the symmetric group on $n$ letters, whose descents only occur at positions in a. That is, $w(i)>w(i+1)$ implies that $i \in\left\{a_{1}, a_{2}, \ldots, a_{r}\right\}$. Let $I_{\mathbf{a}}$ be this set of indices. For a complete flag $F_{\boldsymbol{\bullet}}$ and $w \in I_{\mathbf{a}}, \mathbb{F} \ell_{\mathbf{a}}$ has a Schubert variety

$$
X_{w} F_{\bullet}:=\left\{E_{\bullet} \in \mathbb{F} \ell_{\mathbf{a}} \mid \operatorname{dim} E_{a_{j}} \cap F_{i} \geq \#\left\{l \leq a_{j} \mid w(l) \leq i\right\}\right\} .
$$

The codimension of $X_{w} F_{\bullet}$ is $\ell(w):=\#\{i<j \mid w(i)>w(j)\}$. We state the general question in the Schubert calculus of enumerative geometry for flags.

Question 4.9. Given permutations $w_{1}, w_{2}, \ldots, w_{s} \in I_{\mathbf{a}}$ with $\ell\left(w_{1}\right)+\ell\left(w_{2}\right)+\cdots+$ $\ell\left(w_{s}\right)=\operatorname{dim} \mathbb{F} \ell_{\mathbf{a}}$ and general flags $F_{\bullet^{1}}{ }^{1}, F_{\bullet}{ }^{2}, \ldots, F_{\bullet}{ }^{s}$, what is the number of points in the intersection

$$
X_{w_{1}} F_{\bullet}^{1} \cap X_{w_{2}} F_{\bullet}^{2} \cap \cdots \cap X_{w_{s}} F_{\bullet}^{s} ?
$$

There are algorithms [2, 13] for computing this number and the numbers for the Lagrangian Schubert calculus in Section 4.2.4. When $w=(i, i+1), X_{w} F_{\bullet}$ is the simple Schubert variety, written $X_{i} F_{\bullet}$,

$$
X_{i} F_{\bullet}=X_{(i, i+1)} F_{\bullet}=\left\{E_{\bullet} \in \mathbb{F} \ell_{n} \mid E_{i} \cap F_{n-i} \neq\{0\}\right\} .
$$

Theorem $4.10\left(\left[75\right.\right.$, Corollary 2.2]). Given a list $i_{1}, i_{2}, \ldots, i_{N}\left(N=\operatorname{dim} \mathbb{F} \ell_{\mathbf{a}}\right)$ of numbers with $i_{j} \in \mathbf{a}$, there exist real flags $F_{\bullet}{ }^{1}, F_{\bullet}{ }^{2}, \ldots, F_{\bullet}{ }^{N}$ such that the intersection of simple Schubert varieties

$$
X_{i_{1}} F_{\bullet}^{1} \cap X_{i_{2}} F_{\bullet}^{2} \cap \cdots \cap X_{i_{N}} F_{\bullet}^{N}
$$

is transverse and consists only of real flags.

The case of $\mathbf{a}=2<n-2$ of this theorem was proven earlier [70, Theorem 13]. It remains open whether the general Schubert calculus of flags is fully real. 
4.2.4. Unreality in the Lagrangian Schubert calculus. Let $V$ be a $2 n$-dimensional vector space equipped with the alternating bilinear form

$$
\left\langle\sum x_{i} e_{i}, \sum y_{j} e_{j}\right\rangle:=\sum_{i=1}^{n} x_{i} y_{2 n+1-i}-y_{i} x_{2 n+1-i} .
$$

A subspace $H \subset V$ is isotropic if the restriction of the form to $H$ is identically zero, $\langle H, H\rangle \equiv 0$. The dimension of an isotropic subspace is at most $n$ and Lagrangian subspaces are isotropic subspaces with this maximal dimension. The Lagrangian Grassmannian $L G(n)$ is the set of Lagrangian subspaces of $V$, an algebraic manifold of dimension $\left(\begin{array}{c}n+1 \\ 2\end{array}\right)$.

A flag $F_{\bullet}$ in $V$ is isotropic if $F_{n}$ is Lagrangian and $\left\langle F_{i}, F_{2 n-i}\right\rangle \equiv 0$ for all $i=$ $1,2, \ldots, 2 n-1$. Given an isotropic flag $F_{\bullet}$, the Lagrangian Grassmannian has Schubert varieties $\Psi_{\lambda} F_{\text {}}$ indexed by decreasing sequences $\lambda: n \geq \lambda_{1}>\lambda_{2}>\cdots>\lambda_{l}>0$ of positive integers, called strict partitions. (Here $l$ can be any integer between 0 and $n$ ). The codimension of $\Psi_{\lambda} F_{\bullet}$ is $|\lambda|=\lambda_{1}+\lambda_{2}+\cdots+\lambda_{l}$. The Lagrangian Schubert calculus asks for the number of points in a transverse zero-dimensional intersection of Schubert varieties.

The simple Schubert variety $\Psi_{1} F_{\bullet}$ consists of those Lagrangian subspaces meeting the Lagrangian subspace $F_{n}$ non-trivially. The simple Lagrangian Schubert calculus is fully unreal.

THEOREM 4.11. There exist isotropic real flags $F_{\bullet}{ }^{1}, F_{\bullet}^{2}, \ldots, F_{\bullet}\left(\begin{array}{c}n+1 \\ 2\end{array}\right)$ such that the intersection of Schubert varieties

$$
\Psi_{1} F_{\bullet}^{1} \cap \Psi_{1} F_{\bullet}^{2} \cap \cdots \cap \Psi_{1} F_{\bullet}^{\left(\begin{array}{c}
n+1 \\
2
\end{array}\right)}
$$

is transverse with no real points.

REMARK 4.12. In [75, Theorem 4.2] flags were given so that the intersection had no real points, and it was not known if that intersection was transverse. Perturbing those flags slightly (so that the intersection becomes transverse) gives the above result.

We do not know if these (or many other) enumerative problems in the Lagrangian

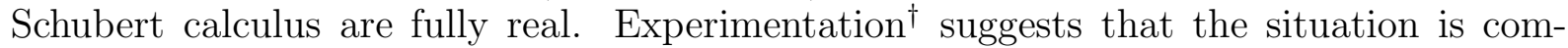
plicated. Briefly, while many other enumerative problems in the Lagrangian Schubert calculus are fully unreal, there are a few which are fully real. For example, there exist 2 real isotropic 2-planes and 2 real isotropic 3-planes such that all 4 Lagrangian subspaces meeting each of these are real (see Theorem 5.13).

The problems of Theorem 4.11 may give examples of enumerative problems that are not fully real. Experimental evidence suggests however that this may be unlikely. For example, (case $n=3$ of Theorem 4.11) there are 16 Lagrangian subspaces in $\mathbb{C}^{6}$ having non-trivial intersection with 6 general Lagrangian subspaces. We computed 30,000 random instances of this enumerative problem, and found several examples of 6 real Lagrangian subspaces such that all 16 Lagrangian subspaces meeting them are real. Table 1 shows the number of these 30,000 systems having a given number of real solutions.

\footnotetext{
${ }^{\dagger}$ This will be reported in the forthcoming article [78].
} 


\begin{tabular}{|c||c|c|c|c|c|c|c|c|c|}
\hline Number of real solutions & 0 & 2 & 4 & 6 & 8 & 10 & 12 & 14 & 16 \\
\hline Frequency & 4983 & 8176 & 9314 & 5027 & 1978 & 445 & 67 & 8 & 2 \\
\hline
\end{tabular}

TABLE 1. Frequency with given number of real solutions.

\section{The Conjecture of Shapiro and Shapiro}

The results of Section 1 were inspired by a remarkable conjecture of Boris Shapiro and Michael Shapiro. Let $\gamma: \mathbb{R} \rightarrow \mathbb{R}^{n}$ be the rational normal curve

$$
\gamma(t)=\left(1, t, t^{2}, \ldots, t^{n-1}\right)=\sum_{i=1}^{n} t^{i-1} e_{i}
$$

For $t \in \mathbb{R}$, define the flag $F_{\bullet}(t)$ by any of the three equivalent ways

$$
\begin{aligned}
F_{i}(t) & =\text { linear span of } \gamma(t), \frac{d}{d t} \gamma(t), \ldots, \frac{d^{i-1}}{d t^{i-1}} \gamma(t), \\
& =\text { row space }\left[\begin{array}{ccccccc}
1 & t & t^{2} & & \cdots & t^{n-1} \\
0 & 1 & 2 t & & \cdots & & (n-1) t^{n-2} \\
\vdots & & \ddots & \ddots & & & \vdots \\
0 & \cdots & 0 & 1 & i t & \cdots & \left(\begin{array}{c}
n-1 \\
i-1
\end{array}\right) t^{n-i}
\end{array}\right], \\
& =i \text {-plane osculating rational normal curve } \gamma \text { at } \gamma(t) .
\end{aligned}
$$

This makes sense for $t \in \mathbb{C}$ and is extended to $t=\infty \in \mathbb{P}^{1}$ by setting $F_{i}(\infty)$ to be the row space of the last $i$ rows of the $n \times n$ identity matrix.

Conjecture 5.1 (Shapiro-Shapiro). Let $\alpha^{1}, \alpha^{2}, \ldots, \alpha^{s} \in\left(\begin{array}{c}{[n]} \\ k\end{array}\right)$ be such that $\left|\alpha^{1}\right|+$ $\left|\alpha^{2}\right|+\ldots+\left|\alpha^{s}\right|=k(n-k)$. Then, for every distinct $t_{1}, t_{2}, \ldots, t_{s} \in \mathbb{R}$, the intersection of Schubert varieties

$$
X_{\alpha^{1}} F_{\bullet}\left(t_{1}\right) \cap X_{\alpha^{2}} F_{\bullet}\left(t_{2}\right) \cap \cdots \cap X_{\alpha^{s}} F_{\bullet}\left(t_{s}\right)
$$

is (a) transverse, and (b) consists only of real points.

Eisenbud's and Harris's dimensional transversality result [15, Theorem 2.3] guarantees that the intersection (5.4) is zero-dimensional. Not only does Conjecture 5.1 state that the classical Schubert calculus is fully real, but it also proposes flags witnessing this full reality. This conjecture has been central to subsequent developments in the real Schubert calculus and it has direct connections to other parts of mathematics, including linear systems theory and linear series on $\mathbb{P}^{1}$ (see Remark 5.8). The article [74] and the web page [71] give a more complete discussion.

One aspect of this conjecture which we relate is the following.

TheOREm 5.2 ([74, Theorem 3.3]). For a given $k$ and $n$, the general case of Conjecture 5.1 follows from the special case when each Schubert condition is simple, that is, when each $\alpha^{i}=(1,2, \ldots, k-1, k+1)$.

Consider these osculating flags $F_{\bullet}(t)$ in more detail. If the $i$ th row of the matrix (5.2) is multiplied by $t^{i}$ (which does not affect its row space when $t \neq 0$ ), then the entry in 
position $i, j$ is $\left(\begin{array}{c}j-1 \\ i-1\end{array}\right) t^{j}$, and so we have

$$
F_{i}(t)=t \cdot F_{i}(0),
$$

where $t . F_{i}(0)$ is given by the action $(4.9)$ of $\mathbb{R}^{\times}$on $\mathbb{R}^{n}$. The $\alpha$ th Plücker coordinate of $F_{i}(0)$ is

$$
p_{\alpha}\left(F_{i}(0)\right)=\prod_{j<l} \frac{\alpha_{j}-\alpha_{l}}{j-l},
$$

which is non-vanishing. Thus Theorem 4.6 has the following corollary.

TheOREM $5.3\left(\mathbb{7 2}\right.$, Theorem 1]). There exist $t_{1}, t_{2}, \ldots, t_{k(n-k)} \in \mathbb{R}$ such that there are exactly $d(k, n) k$-planes meeting each $(n-k)$-plane $F_{n-k}\left(t_{i}\right)$ non-trivially, and all are real. Equivalently, if $\alpha=(1,2, \ldots, k-1, k+1)$ so that $|\alpha|=1$, then the intersection of Schubert varieties

$$
X_{\alpha} F_{\bullet}\left(t_{1}\right) \cap X_{\alpha} F_{\bullet}\left(t_{2}\right) \cap \cdots \cap X_{\alpha} F_{\bullet}\left(t_{k(n-k)}\right)
$$

is transverse with all points real.

This establishes a weak form of Conjecture 5.1 for simple Schubert conditions, replacing the quantifier for all $t_{i} \in \mathbb{R}$ by there exists $t_{i} \in \mathbb{R}$.

If the parameters $t_{i}$ in (5.6) vary, then the number of real points in that intersection could change, but only if two points first collide (prior to spawning a complex conjugate pair of solutions). This is the reverse of the progression in Dietmaier's algorithm, as displayed in Figure 6. This situation cannot occur if the intersection remains transverse. Together with Theorems 5.2 and 5.3 we deduce the following result.

Theorem 5.4 ( $[\mathbf{7 2}$, Theorem 6]). Part (a) of Conjecture [5.1 for simple conditions implies part (b) for arbitrary Schubert conditions.

If $t>0$, then by (5.5) the Plücker coordinates of $F_{i}(t)$ are strictly positive. An upper triangular $n \times n$ matrix $g$ is totally positive if every $i \times i$ subdeterminant of $g$ is non-negative, and vanishes only if that subdeterminant vanishes on all upper triangular matrices. For example, when $t>0$ and $i=n$, the matrix (5.2) is totally positive. Write $\Gamma(t)$ for this matrix. It has the form $e^{t \eta}$ where $\eta$ is the principal nilpotent matrix $\Gamma^{\prime}(0)$. Observe that if $t_{1}<t_{2}<\cdots<t_{s}$, then

$$
F_{\bullet}\left(t_{i}\right)=\Gamma\left(t_{i}-t_{i-1}\right) \cdot F_{\bullet}\left(t_{i-1}\right)
$$

Conjecture 5.1 has a more general version involving totally positive matrices.

Conjecture 5.5 (Shapiro-Shapiro [74, Conjecture 4.1]). Let $\alpha^{1}, \alpha^{2}, \ldots, \alpha^{s} \in\left(\begin{array}{c}{[n]} \\ k\end{array}\right)$ be such that $\left|\alpha^{1}\right|+\left|\alpha^{2}\right|+\ldots+\left|\alpha^{s}\right|=k(n-k)$ and suppose $g_{2}, g_{3}, \ldots, g_{s}$ are totally positive matrices. Given any real flag $F_{\bullet}{ }^{1}$, define $F_{\bullet}{ }^{i}$ for $i>1$ by $F_{\bullet}{ }^{i}:=g_{i} \cdot F_{\bullet}{ }^{i-1}$, the intersection of Schubert varieties

$$
X_{\alpha^{1}} F_{\bullet}^{1} \cap X_{\alpha^{2}} F_{\bullet}^{2} \cap \cdots \cap X_{\alpha^{s}} F_{\bullet}^{s}
$$

is transverse with all points real.

There is some experimental evidence for this version of Conjecture 5.1. Subsequent conjectures involving osculating flags have versions involving totally positive matrices. We leave their statements to the reader, they will be explored more fully in [78]. 
5.1. Rational functions with real critical points. By far the strongest evidence for Conjecture 5.1 is that it is true when $k$ or $n-k$ is equal to 2 .

TheOREm 5.6 (Eremenko and Gabrielov [16]). Conjecture 5.1 is true when one of $k$ or $n-k$ is 2 .

This is a consequence of a theorem about rational functions with real critical points. A rational function is an algebraic map $\varphi: \mathbb{P}^{1} \rightarrow \mathbb{P}^{1}$. Two rational functions $\varphi_{1}$ and $\varphi_{2}$ are equivalent if $\varphi_{1}=g \circ \varphi_{2}$, where $g$ is a fractional linear transformation.

THEOREM 5.7 ([16]). If all the critical points of a rational function are real, then it is equivalent to a real rational function.

Consider the composition

$$
\mathbb{P}^{1} \longrightarrow \mathbb{P}^{d}-\rightarrow \mathbb{P}^{1}
$$

where the first map is the rational normal curve

$$
\gamma:[s, t] \longmapsto\left[s^{d}, s^{d-1} t, \ldots, s t^{d-1}, t^{d}\right]
$$

and the second is a linear projection

$$
\left[x_{0}, x_{1}, \ldots, x_{d}\right] \longmapsto\left[\Lambda_{1}(x), \Lambda_{2}(x)\right]
$$

where $\Lambda_{1}$ and $\Lambda_{2}$ are independent linear forms. Let $E \subset \mathbb{P}^{d}$ be the center of this projection, the linear subspace where $\Lambda_{1}=\Lambda_{2}=0$. When $E$ is disjoint from the rational normal curve, this composition defines a rational function of degree $d$, and all such rational functions occur in this manner. In fact, equivalence classes of rational maps are exactly those maps with the same center of projection. A rational function $\varphi$ has a critical point at $t \in \mathbb{P}^{1}$ if $d \varphi$ vanishes at $t$. If we consider the composition (5.7), this implies that the center $E$ meets the line tangent to the rational normal curve $\gamma\left(\mathbb{P}^{1}\right)$ at $\gamma(t)$.

Goldberg 24 asked (and answered) the question: how many equivalence classes of rational functions of degree $d$ have a given set of $2 d-2$ critical points? Reasoning as above, she reduced this to the problem of determining the number of codimension 2 planes $E$ in $\mathbb{P}^{d}$ meet $2 d-2$ given tangents to the rational normal curve. Formulating this in the dual projective space, we recover the problem of the Introduction to Section 4 : Determine the number of lines in $\mathbb{P}^{d}$ meeting $2 d-2$ general codimension 2 planes. The answer is the $d$ th Catalan number, $C_{d}=\frac{1}{d}\left(\begin{array}{c}2 d-1 \\ d-1\end{array}\right)(4.1)$.

Consider the above description in the affine cone over $\mathbb{P}^{d}$. The centers $E \in \operatorname{Gr}(d-1, d+1)$ giving a rational function of degree $d$ with critical point at $t \in \mathbb{C} \subset \mathbb{P}^{1}$ are points in the Schubert variety $X_{\alpha} F_{\bullet}(t)$, where $\alpha$ is the simple Schubert condition of Theorem 5.2 and $F_{\bullet}(t)$ is the flag of subspaces osculating the rational normal curve $\gamma\left(\mathbb{P}^{1}\right)$ at $\gamma(t)$. An equivalence class of rational functions contains a real rational function when the common center $E$ is real. In this way, Theorem 5.7 implies Conjecture 5.1 when $n-k=2$ and each $\left|\alpha^{i}\right|=1$. By Theorem 5.2, this implies the full conjecture when $n-k=2$. Working in the dual space, we deduce Conjecture 5.1 when $k=2$.

Gabrielov and Eremenko prove Theorem 5.7 by showing there exist $C_{d}$ distinct real rational functions with critical points at a given set of $2 d-2$ real numbers. Let $\varphi: \mathbb{P}^{1} \rightarrow \mathbb{P}^{1}$ be a real rational function of degree $d$ with only real critical points. Then $\varphi^{-1}\left(\mathbb{P}_{\mathbb{R}}^{1}\right) \subset \mathbb{P}_{\mathbb{C}}^{1}$ is an embedded graph containing $\mathbb{P}_{\mathbb{R}}^{1}$ which is stable under complex conjugation and whose vertices have valence 4 and are at the critical points. There are exactly $C_{d}$ isotopy classes of such graphs. For each isotopy class $\Gamma$ and collection of $2 d-2$ distinct real points, 
they essentially construct a rational function $\varphi$ of degree $d$ having these critical points with $\varphi^{-1}\left(\mathbb{P}_{\mathbb{R}}^{1}\right) \in \Gamma$. A key point involves degenerate rational maps with fewer than $2 d-2$ critical points. It may be interesting to relate this to the degenerations in the proof of Theorem 4.6.

REMARK 5.8. If we project the rational normal curve from a center $E$ of codimension $m+1$, then the image of the projection is $\mathbb{P}^{m}$ and the composition is a parameterized rational curve in $\mathbb{P}^{m}$. Post-composition by an element of Aut $\left(\mathbb{P}^{m}\right)$ defines an equivalence relation on such maps and equivalence classes are determined by the centers of projection. These centers are codimension $m+1$ linear series of degree $d$ on $\mathbb{P}^{1}$. Such a linear series is ramified at a point $t \in \mathbb{P}^{1}$ when the center $E$ meets the $m$-plane osculating the rational normal curve. A rational curve/linear series is maximally inflected if the ramification is at real points. We just considered the case $d=1$ of rational functions with real critical points, and the case $d=2$ was discussed in Section 3.1. The existence of maximally inflected curves with simple ramification is a consequence of Theorem 5.3, and Conjecture 5.1 predicts this is a rich class of real curves in $\mathbb{P}^{m}$. This connection between linear series and the Schubert calculus originated in work of Castelnuovo [7].

5.2. Generalizations of Conjecture 5.1. The Grassmannian, flag manifolds, and Lagrangian Grassmannian are examples of flag varieties $G / P$ where $G$ is a reductive algebraic group and $P$ a parabolic subgroup. These flag varieties have Schubert varieties and the most general form of the Schubert calculus involves zero-dimensional intersections of these Schubert varieties. Likewise, these flag varieties have real forms (given by split ${ }^{\dagger}$ forms of $G$ and $P$ ) and there is a generalization of Conjecture 5.1 for these real forms. This generalization is false, but in a very interesting way. We describe what is known about this general conjecture for the flag manifolds and the Lagrangian Grassmannian, and give conjectures describing what we believe to be true.

5.2.1. The manifold of partial flags. Let $\mathbf{a}:=0<a_{1}<a_{2}<\cdots<a_{r}<a_{r+1}=n$ be a sequence of integers. The straightforward generalization (which was its original form and which is false) of Conjecture 5.1 for the flag manifold $\mathbb{F} \ell_{\mathbf{a}}$ declares that a zero-dimensional intersection of Schubert varieties given by flags osculating the real rational normal curve consists only of real points.

For $\alpha \in\left(\begin{array}{c}{[n]} \\ a_{i}\end{array}\right)$, we have the Grassmannian Schubert variety

$$
Y_{\alpha, i} F_{\bullet}:=\left\{E_{\bullet} \in \mathbb{F} \ell_{\mathbf{a}} \mid E_{a_{i}} \in X_{\alpha} F_{\bullet} \subset \operatorname{Gr}\left(a_{i}, n\right)\right\} .
$$

For example, if $|\alpha|=1$, then $Y_{\alpha, i} F_{\bullet}$ is the simple Schubert variety $X_{i} F_{\bullet}$ of Section 4.2 .3 .

EXAMPLE 5.9. Let $\mathbf{a}=2<3<5=n$ so that $\mathbb{F} \ell_{\mathbf{a}}$ is the manifold of partial flags $E_{2} \subset E_{3}$ in 5-space. This flag manifold has two simple Schubert varieties

$$
\begin{aligned}
& X_{2} F_{\bullet}=\left\{E_{2} \subset E_{3} \mid E_{2} \cap F_{3} \neq\{0\}\right\} \\
& X_{3} F_{\bullet}=\left\{E_{2} \subset E_{3} \mid E_{3} \cap F_{2} \neq\{0\}\right\}
\end{aligned}
$$

A calculation [75, Example 2.5] shows that

$$
\begin{gathered}
X_{2} F_{\bullet}(-8) \cap X_{3} F_{\bullet}(-4) \cap X_{2} F_{\bullet}(-2) \cap X_{3} F_{\bullet}(-1) \cap \\
X_{2} F_{\bullet}(1) \cap X_{3} F_{\bullet}(2) \cap X_{2} F_{\bullet}(4) \cap X_{3} F_{\bullet}(8)
\end{gathered}
$$

is transverse with none of its 12 points real.

\footnotetext{
${ }^{\dagger}$ Split is a technical term: $\mathbb{R}^{\times} \subset \mathbb{C}^{\times}$is a split form of $G L_{1}$, but $S^{1} \subset \mathbb{C}^{\times}$is not.
} 
Thus the straightforward generalization of Conjecture 5.1 is completely false. On the other hand, if $t_{1}<t_{2}<\cdots<t_{8}$ are any of the 24,310 subsets of eight numbers from

$$
\{-6,-5,-4,-3,-2,-1,1,2,3,5,7,11,13,17,19,23,29\},
$$

then the intersection

$$
\begin{array}{r}
X_{2} F_{\bullet}\left(t_{1}\right) \cap X_{2} F_{\bullet}\left(t_{2}\right) \cap X_{2} F_{\bullet}\left(t_{3}\right) \cap X_{2} F_{\bullet}\left(t_{4}\right) \\
\cap X_{3} F_{\bullet}\left(t_{5}\right) \cap X_{3} F_{\bullet}\left(t_{6}\right) \cap X_{3} F_{\bullet}\left(t_{7}\right) \cap X_{3} F_{\bullet}\left(t_{8}\right)
\end{array}
$$

is transverse with all of its 12 points real [75, Example 2.5].

We have used more than $2 \times 10^{7}$ seconds of CPU time investigating this problem of intersections of Schubert varieties in manifolds of partial flags given by flags osculating the rational normal curve, and a picture is emerging of what to expect, at least for Grassmannian Schubert varieties.

Suppose we have a list of indices of Grassmannian Schubert varieties

$$
\left(\alpha^{1}, i_{1}\right),\left(\alpha^{2}, i_{2}\right), \ldots,\left(\alpha^{s}, i_{s}\right), \quad \text { with } \quad \alpha^{j} \in\left(\begin{array}{c}
{[n]} \\
i_{j}
\end{array}\right)
$$

where $i_{j} \in\left\{a_{1}, \ldots, a_{r}\right\}$ and $\left|\alpha^{1}\right|+\left|\alpha^{2}\right|+\cdots+\left|\alpha^{s}\right|=\operatorname{dim} \mathbb{F} \ell_{\mathbf{a}}$. Call such a list Grassmannian Schubert data for $\mathbb{F} \ell_{\mathbf{a}}$. Consider an intersection of Grassmannian Schubert varieties

$$
Y_{\alpha^{1}, i_{1}} F_{\bullet}\left(t_{1}\right) \cap Y_{\alpha^{2}, i_{2}} F_{\bullet}\left(t_{2}\right) \cap \cdots \cap Y_{\alpha^{s}, i_{s}} F_{\bullet}\left(t_{s}\right),
$$

where $t_{1}<t_{2}<\cdots<t_{s}$ are distinct real numbers and $F_{\bullet}(t)$ is the flag osculating the rational normal curve $\gamma$ at $\gamma(t)$.

Conjecture 5.10. Let $\mathbf{a}=1<a_{1}<a_{2}<\cdots<a_{r}<a_{r+1}=n$ and

$$
\left(\alpha^{1}, i_{1}\right),\left(\alpha^{2}, i_{2}\right), \ldots,\left(\alpha^{s}, i_{s}\right), \quad \text { with } \quad \alpha^{j} \in\left(\begin{array}{c}
{[n]} \\
i_{j}
\end{array}\right)
$$

be Grassmannian Schubert data for $\mathbb{F} \ell_{\mathbf{a}}$.

1. For every choice of real numbers $t_{1}<t_{2}<\cdots<t_{s}$, the intersection (5.10) is (a) transverse with (b) with all points of intersection real if the indices $i_{j}$ are in order: $i_{1} \leq i_{2} \leq \cdots \leq i_{s}$ or else $i_{1} \geq i_{2} \geq \cdots \geq i_{s}$.

2. If the indices $i_{j}$ are not in order, nor is any cyclis permutation, then there exist real numbers $t_{1}<t_{2}<\cdots<t_{s}$ such that the intersection (5.10) is transverse with all points real, and there exist real numbers $t_{1}<t_{2}<\cdots<t_{s}$ such that the intersection is transverse with not all points real.

In particular, enumerative problems involving Grassmannian Schubert varieties on $\mathbb{F} \ell_{\mathbf{a}}$ are fully real.

\section{REMARK 5.11.}

1. When each $\left|\alpha^{i}\right|=1$, there are real numbers $t_{1}<t_{2}<\ldots<t_{s}$ such that the intersection (5.10) is transverse with all points real $\mathbf{7 5}$, Corollary 2.2]. Thus the number of real points in the intersection (5.10) is expected to vary when the the indices $i_{j}$ are not in order.

2. When $r=1, \mathbb{F} \ell_{\mathbf{a}}$ is the Grassmannian $\operatorname{Gr}\left(a_{1}, n\right)$ and the condition on the indices being ordered is empty, so this case of Conjecture 5.10 reduces to Conjecture 5.1. 
3. There is considerable evidence for this conjecture when $r=2$. Part (2) is true for every set of Grassmannian Schubert data in $\mathbb{F} \ell_{2<3} \mathbb{C}^{5}$ and all except one such set in $\mathbb{F} \ell_{2<4} \mathbb{C}^{6 \dagger}$. Many instances of these same enumerative problems with ordered partitions have been computed, and in each instance of (1) the intersection (5.10) is transverse with all points real.

4. As for Conjecture 5.1, (a) implies (b) in Part (1) of Conjecture 5.10.

5. We have tested no instances of the intersection (5.10) with $r>2$, so the truth may differ from the exact statement of Conjecture 5.10.

6. Conjecture 5.10 has nothing to say when the Schubert data are not Grassmannian.

5.2.2. The Lagrangian Grassmannian. As in Section 4.2.4, let $V$ be a $2 n$-dimensional vector space equipped with the alternating form $\langle\cdot, \cdot\rangle$ defined by (4.18). Set

$$
\gamma(t):=\left(1, t, \frac{t^{2}}{2}, \ldots, \frac{t^{n}}{n !},-\frac{t^{n+1}}{(n+1) !}, \frac{t^{n+2}}{(n+2) !}, \ldots,(-1)^{n-1} \frac{t^{2 n-1}}{(2 n-1) !}\right) .
$$

The flag $F_{\bullet}(t)$ of subspaces osculating this rational normal curve is isotropic for all $t \in \mathbb{C}$.

Given a strict partition $\lambda: \lambda_{1}>\lambda_{2}>\cdots>\lambda_{l}>0$ and an isotropic flag $F_{\bullet}$, set

$$
\Psi_{\lambda} F_{\bullet}:=\left\{H \in L G(n) \mid H \cap F_{n+1-\lambda_{i}} \geq i \text { for } i=1, \ldots, l\right\} .
$$

The codimension of this Schubert variety is $|\lambda|=\lambda_{1}+\lambda_{2}+\cdots+\lambda_{l}$. For example,

$$
\Psi_{a} F_{\bullet}=\left\{H \mid H \cap F_{n+1-a} \neq\{0\}\right\} .
$$

With these definitions, we state the version of Theorem 4.11 proven in 475 .

TheOrem $5.12\left(\llbracket 75\right.$, Theorem 4.2]). Given a strict partition $\lambda$, let $N:=\left(\begin{array}{c}n+1 \\ 2\end{array}\right)-|\lambda|=$ $\operatorname{dim} \Psi_{\lambda} F_{\bullet}$. If $N>1$, then there exist distinct real numbers $t_{1}, t_{2}, \ldots, t_{N}$ such that

$$
\Psi_{\lambda} F_{\bullet}(0) \cap \Psi_{1} F_{\bullet}\left(t_{1}\right) \cap \Psi_{1} F_{\bullet}\left(t_{2}\right) \cap \cdots \cap \Psi_{1} F_{\bullet}\left(t_{N}\right)
$$

is zero-dimensional with no points real.

Thus the generalization of Conjecture 5.1 is badly false for the Lagrangian Grassmannian. On the other hand it holds for some enumerative problems in the Lagrangian Grassmannian.

TheOREM $5.13(\| 78)$. For any distinct real numbers $t_{1}, t_{2}, t_{3}, t_{4}$, the intersection of Schubert varieties in $\overline{L G}(3)$

$$
\Psi_{1} F_{\bullet}\left(t_{1}\right) \cap \Psi_{1} F_{\bullet}\left(t_{2}\right) \cap \Psi_{2} F_{\bullet}\left(t_{3}\right) \cap \Psi_{2} F_{\bullet}\left(t_{4}\right)
$$

is transverse with all points real.

These two results illustrate a dichotomy that is emerging from experimentation. Call a list of strict partitions $\lambda^{1}, \lambda^{2}, \ldots, \lambda^{s}$ with $\left|\lambda^{1}\right|+\left|\lambda^{2}\right|+\cdots+\left|\lambda^{s}\right|=\left(\begin{array}{c}n+1 \\ 2\end{array}\right)$ Lagrangian Schubert data. In every instance we have computed of a zero-dimensional intersection of Lagrangian Schubert varieties whose flags osculate the rational normal curve (5.11), the intersection has been transverse with either all points real or no points real. Most interestingly, the outcome - all real or no real-has depended only upon the Lagrangian Schubert data of the intersection.

${ }^{\dagger}$ The one exception is due to our inability to compute any instances. 
Conjecture 5.14. Given Lagrangian Schubert data $\lambda^{1}, \lambda^{2}, \ldots, \lambda^{s}$ and distinct real numbers $t_{1}, t_{2}, \ldots, t_{s}$, the intersection

$$
\Psi_{\lambda^{1}} F_{\bullet}\left(t_{1}\right) \cap \Psi_{2} \lambda^{2} F_{\bullet}\left(t_{2}\right) \cap \cdots \cap \Psi_{\lambda^{s}} F_{\bullet}\left(t_{s}\right)
$$

is transverse with either

(a) all points real, or

(b) no points real,

and the outcome (a) or (b) depends only upon the list $\lambda^{1}, \lambda^{2}, \ldots, \lambda^{s}$.

We do not have a good idea what distinguishes the Lagrangian Schubert data giving all points real from the data giving no points real. Further experimentation is needed.

5.2.3. Further generalizations of Conjecture 5.1. The status of the generalizations of Conjecture 5.1 to other flag varieties is almost completely unknown. There is one flag variety, the Orthogonal Grassmannian, for which much is known. In particular, the analog of Theorem 5.3 (involving codimension 1 Schubert varieties given by isotropic flags osculating the rational normal curve) holds for the Orthogonal Grassmannian [75, Corollary 3.3]. There has also been a significant amount of computer experimentation testing cases of the obvious generalization of Conjecture 5.1 for the orthogonal Grassmannian, and in each, all points of intersection were found to be real. Lastly, we remark that we do not know of any reasonable version of Conjecture 5.1 for the quantum Schubert calculus.

\section{Lower Bounds in the Schubert calculus}

In Section 3.3, we saw that of the 12 rational cubics meeting 8 real points in the plane, at least 8 were real. This was the first instance of a non-trivial lower bound on the number of real solutions to a problem in enumerative geometry. Recent work of Eremenko and Gabrielov shows this phenomenon is pervasive in the Schubert calculus.

Recall from Section 4 that given general codimension 2 planes $L_{1}, L_{2}, \ldots, L_{2 n-2}$ in $\mathbb{P}^{n}$, there are exactly $C_{n}=\frac{1}{n}\left(\begin{array}{c}2 n-2 \\ n-1\end{array}\right)$ lines meeting each $L_{i}$. For each $n \geq 2$, define

$$
u_{n}:=\left\{\begin{array}{ll}
0 & \text { if } n \text { is odd } \\
C_{n / 2} & \text { if } n \text { is even }
\end{array} .\right.
$$

Theorem 6.1 (Eremenko and Gabrielov [19, Corollary 1.5]). Given general codimension 2 planes $L_{1}, L_{2}, \ldots, L_{2 n-2}$ in $\mathbb{P}_{\mathbb{R}}^{n}$, the number $\rho$ of real lines $\ell$ meeting each $L_{i}$ satisfies

$$
u_{n} \leq \rho \leq C_{n} \text {. }
$$

\begin{tabular}{|c||c|c|c|c|c|c|c|c|c|c|c|}
\hline$n$ & 2 & 3 & 4 & 5 & 6 & 7 & 8 & 9 & 10 & 11 & 12 \\
\hline$C_{n}$ & 1 & 2 & 5 & 14 & 42 & 132 & 429 & 1430 & 4862 & 16796 & 58786 \\
\hline$u_{n}$ & 1 & 0 & 1 & 0 & 2 & 0 & 5 & 0 & 14 & 0 & 42 \\
\hline
\end{tabular}

TABLE 2. Upper and lower bounds for the Schubert calculus of lines

We display these bounds for small $n$ in Table 2. The sharpness of the upper bound is provided by Theorem 4.2. The sharpness of the lower bound remains open. The lower bounds are sharp, if we allow real conditions of the form: the line meets two complex conjugate codimension 2 planes. When $n$ is odd so that $u_{n}=0$, this gives an important result for the problem of placing real poles in linear systems theory [18]. The existence of 
a lower bound comes from new ideas involving the degree of a linear projection of a real Grassmannian.

Recall from Section 4.1 that the $k$-planes in $\mathbb{C}^{n}$ meeting $k(n-k)$ general $(n-k)$-planes non-trivially is a complementary dimensional linear section of the Grassmannian,

$$
\Lambda \cap \operatorname{Gr}(k, n) \text {, }
$$

where $\Lambda$ has codimension $k(n-k)$ in Plücker space. The number of such $k$-planes is the degree of $\operatorname{Gr}(k, n)$. This is also the degree of the linear projection

$$
\pi_{E}: \operatorname{Gr}(k, n) \hookrightarrow \mathbb{P}^{\left(\begin{array}{l}
n \\
k
\end{array}\right)-1} \rightarrow \mathbb{P}^{k(n-k)}
$$

with center of projection a plane $E$ with codimension $k(n-k)+1$ disjoint from $\operatorname{Gr}(k, n)$. The connection between these two definitions of degree is that when $E \subset \Lambda, \pi_{E}(\Lambda)$ is a point $x \in \mathbb{P}^{k(n-k)}$ and

$$
\Lambda \cap \operatorname{Gr}(k, n)=\pi_{E}^{-1}(x) .
$$

Since complex manifolds are canonically oriented, the degree of such a linear projection is just the number of points in the inverse image of a regular value $x$.

An important such linear projection is the Wronski map. Let $L(t)$ be the $(n-k)$ plane osculating the rational normal curve (5.1). By (4.6) and the discussion following Theorem 5.2, the equation for a $k$-plane $K$ to meet $L(t)$ non-trivially is

$$
\sum t^{\left(\begin{array}{c}
n+1 \\
2
\end{array}\right)-\left(\begin{array}{c}
n-k+1 \\
2
\end{array}\right)-|\alpha|} L_{\alpha} p_{\alpha}(K)
$$

where $p_{\alpha}(K)$ is the $\alpha$ th Plücker coordinate of $K$ and $L_{\alpha}$ is the appropriately signed Plücker coordinate of $L(0)$ complementary to $\alpha$. The association of a $k$-plane $K$ to the polynomial (6.1) is the Wronski map

$$
\pi_{W}: \operatorname{Gr}(k, n) \longrightarrow \mathbb{P}^{k(n-k)},
$$

where $\mathbb{P}^{k(n-k)}$ is the space of polynomials of degree at most $k(n-k)$, modulo scalars. This is a linear projection as the coefficients in (6.1) are linear in the Plücker coordinates. If $f$ has distinct roots $t_{1}, t_{2}, \ldots, t_{k(n-k)}$, then $\pi_{W}^{-1}(f)$ is the set of $k$-planes meeting each of $L\left(t_{1}\right), L\left(t_{2}\right), \ldots, L\left(t_{k(n-k)}\right)$ non-trivially.

To see why this is called the Wronski map, consider $\operatorname{Gr}(k, n)$ as the set of $k$-planes in the space of polynomials of degree at most $n-1$. Given a $k$-plane

$$
K=\text { linear } \operatorname{span}\left\{f_{1}, f_{2}, \ldots, f_{k}\right\},
$$

the Wronski determinant of $K$ is

$$
W(K)=\operatorname{det}\left[\begin{array}{cccc}
f_{1} & f_{2} & \cdots & f_{k} \\
f_{1}^{\prime} & f_{2}^{\prime} & \cdots & f_{k}^{\prime} \\
\vdots & \vdots & \ddots & \vdots \\
f_{1}^{(k-1)} & f_{2}^{(k-1)} & \cdots & f_{k}^{(k-1)}
\end{array}\right]
$$

a polynomial of degree at most $k(n-k)$, well-defined modulo scalars. Under a choice of coordinates given by the coefficients of a polynomial, $W(K)=\pi_{W}(K)$.

Since real manifolds are not necessarily orientable, the degree of a map is a $\mathbb{Z} / 2 \mathbb{Z}$-valued invariant. However, Kronecker [46] defined the degree of a regular map $\mathbb{P}_{\mathbb{R}}^{2} \rightarrow \mathbb{P}_{\mathbb{R}}^{2}$, which he called the characteristic, and his definition makes sense for many maps $f: X \rightarrow Y$ of (not necessarily orientable) compact manifolds. 
First suppose that $X$ is oriented. For a regular value $y \in Y$ of $f$, define

$$
\operatorname{char} f:=\left|\sum_{x \in f^{-1}(y)} \operatorname{sgn} \operatorname{det} d f_{x}\right|,
$$

using local coordinates in $X$ consistent with its orientation and any local coordinate near $y \in Y$. The sum is well-defined up to multiplication by \pm 1 . This number is independent of choices, if $Y$ is connected.

When $X$ may not be orientable, let $\tilde{X} \rightarrow X$ and $\tilde{Y} \rightarrow Y$ be the canonical 2 to 1 coverings of $X$ and $Y$, which have covering group $\mathbb{Z} / 2 \mathbb{Z}$. These are the spaces of orderings of $X$ and $Y$ and are canonically oriented. The map $f$ is orientable if it has a lift $\widetilde{f}: \widetilde{X} \rightarrow \widetilde{Y}$ that is equivariant with respect to the covering group $\mathbb{Z} / 2 \mathbb{Z}$. Define the characteristic of an orientable map $f$ to be char $\widetilde{f}$. This is well-defined, even if $\widetilde{Y}$ consists of 2 components. This characteristic satisfies a fundamental inequality.

Proposition 6.2. Let $f: X \rightarrow Y$ be an orientable map. Then, for every regular value $y \in Y$,

$$
\# f^{-1}(y) \geq \operatorname{char} f .
$$

The fundamental result for this notion of characteristic establishes the existence of a real degree for many even-dimensional varieties.

Theorem 6.3 (Eremenko and Gabrielov [17, Theorem 1.1]). Suppose $X \subset \mathbb{P}_{\mathbb{R}}^{n}$ is a smooth projective variety of even dimension $m$. If there exists a plane $E$ of codimension $m+1$ disjoint from $X$ such that the projection $\pi_{E}: X \longrightarrow \mathbb{P}_{\mathbb{R}}^{m}$ is orientable, then every such projection is orientable, and all have the same characteristic.

We call this the common characteristic the real degree of $X$. When $m$ is odd, the degree of such a projection does depend on the choice of center $E$.

Consider this notion for the real Grassmannian $\mathrm{Gr}_{\mathbb{R}}(k, n)$ which has dimension $k(n-k)$ and is orientable if and only if $n$ is even. When $n$ is even and $k$ is odd, Theorem 6.3 does not apply as $\operatorname{Gr}_{\mathbb{R}}(k, n)$ has odd dimension and when both $n$ and $k$ are even, the characteristic of any projection is defined, as $\operatorname{Gr}_{\mathbb{R}}(k, n)$ is orientable, but it is zero as $\mathbb{P}_{\mathbb{R}}^{k(n-k)}$ is not orientable.

Theorem 6.4 (Eremenko and Gabrielov [17, Theorem 1.2]). When $n$ is odd, any linear projection

$$
\pi_{E}: \operatorname{Gr}_{\mathbb{R}}(k, n) \longrightarrow \mathbb{P}_{\mathbb{R}}^{k(n-k)}
$$

is orientable with characteristic independent of center of projection E.

Eremenko and Gabrielov compute the degree of the Wronski map, giving the real degrees of Grassmannians $\operatorname{Gr}_{\mathbb{R}}(k, n)$ when $n$ is even. To state their result, we introduce some additional combinatorics. Recall the interpretation (4.8)

$$
\text { degree } \operatorname{Gr}(k, n)=\text { \# chains in Bruhat order from } \hat{0} \text { to } \hat{1} \text {, }
$$

where $\hat{1}=(n-k+1, \ldots, n-1, n)$ is the top element in the Bruhat order. We introduce a statistic on these chains. Each cover $\alpha \lessdot \beta$ in the Bruhat order has a unique index $i$ with

$$
\alpha_{i}<\beta_{i}=\alpha_{i}+1 \quad \text { but } \quad \alpha_{j}=\beta_{j} \text { for } j \neq i .
$$


The word $w(\mathrm{ч})$ of a chain ч: $\hat{0} \lessdot \alpha^{1} \lessdot \alpha^{2} \lessdot \cdots \lessdot \alpha^{k(n-k)}=\hat{1}$ is the sequence of indices $\left(i_{1}, i_{2}, \ldots, i_{k(n-k)}\right)$ of these indices for covers in ч. An inversion in such a word is a pair $j<l$ with $i_{j}<i_{l}$, and the weight, $\omega(\mathrm{u})$ is the number of inversions in the word of the chain ч. For instance the chain in the Bruhat order of $\operatorname{Gr}(3,6)$ highlighted in Figure 11

$$
123 \lessdot 124 \lessdot 125 \lessdot 135 \lessdot 145 \lessdot 245 \lessdot 246 \lessdot 346 \lessdot 356 \lessdot 456,
$$

has word 332213121 and length 5 . Define the inversion polynomial

$$
I(k, n)(q):=\sum q^{\omega(\mathrm{ч})},
$$

the sum over all chains $\mathrm{u}$ in the Bruhat order from $\hat{0}$ to $\hat{1}$.

TheOREM 6.5 (Eremenko and Gabrielov [17, Theorem 1.8]). The characteristic of the Wronski map $\pi_{W}: \operatorname{Gr}_{\mathbb{R}}(k, n) \rightarrow \mathbb{P}_{\mathbb{R}}^{k(n-k)}$ is $|I(k, n)(-1)|$.

They prove this by an induction reminiscent of that used in the proof of Theorem 4.6. In the course of their proof, they construct a polynomial $f$ with all roots real having $d(k, n)$ (=degree of the Grassmannian) real points in $\pi_{W}^{-1}(f)$ (one for each chain in the Bruhat order) and show that

$$
\operatorname{det} d \pi_{W}=(-1)^{\omega(\mathrm{u})},
$$

at the point in $\pi_{W}^{-1}(f)$ corresponding to the chain ч.

White [87] studied the statistic $|I(k, n)(-1)|$ and showed that it equals zero if and only if $n$ is even, and that $|I(2,2 n)(-1)|=C_{n}=u_{2 n}$. Eremenko and Gabrielov deduced

Corollary 6.6 (Eremenko and Gabrielov [17, Corollary 1.6]). Given general codimension $(n-k)$-planes $L_{1}, L_{2}, \ldots, L_{k(n-k)}$ in $\mathbb{R}^{n}$, the number $\rho$ of real $k$-planes $K$ meeting each $L_{i}$ non-trivially satisfies

$$
|I(k, n)(-1)| \leq \rho \leq I(n, k)(1) .
$$

In particular, when $n$ is odd this number $\rho$ is non-zero.

These new ideas of Eremenko and Gabrielov, particularly their notion of real degree, greatly increase our understanding of the real Schubert calculus. We emphasize that this is an important start in the search for lower bounds to other enumerative problems. Not all enumerative problems involve a linear projection of a submanifold of projective space. In fact, of the other enumerative problems we have considered in the Schubert calculus, only those of Theorem 4.11 on the Lagrangian Grassmannian involve linear projections, and they have a lower bound of 0 . There has been very little experimental work investigating lower bounds. We describe some of it in the final section.

6.1. Lower bounds in the Schubert calculus for flags? Conjecture 5.10 speculates that a zero-dimensional intersection of Grassmannian Schubert varieties in a flag manifold $\mathbb{F} \ell_{\mathbf{a}}$ has only real points, when the Schubert varieties are given by flags osculating the rational normal curve at points and when the indices of the Grassmannian Schubet data are ordered. If the indices cannot be ordered, then we conjectured (and have found experimentally) that there is a selection of osculating flags with all points of intersection real, as well as a selection with not all points real.

Table 6.1 shows the results of computing 160,000 instances of the intersection of 4 simple Schubert varieties $X_{2} F_{\bullet}\left(t_{i}\right)$ and 4 simple Schubert varieties $X_{3} F_{\bullet}\left(t_{i}\right)$ in the manifold $\mathbb{F} \ell_{2<3}$ of partial flags $E_{2} \subset E_{3}$ in 5-space. Here the numbers $t_{1}<t_{2}<\cdots<t_{8}$ were 20,000 


\begin{tabular}{|c||c|c|c|c|c|c|c|}
\hline \multicolumn{1}{|c||}{ Necklace } & \multicolumn{7}{c|}{ Number of Real Solutions } \\
\cline { 2 - 8 } & 0 & 2 & 4 & 6 & 8 & 10 & 12 \\
\hline \hline 22223333 & 0 & 0 & 0 & 0 & 0 & 0 & 20000 \\
\hline 22322333 & 0 & 0 & 9 & 1677 & 3835 & 6247 & 8232 \\
\hline 22233233 & 0 & 0 & 67 & 3015 & 6683 & 4822 & 5413 \\
\hline 22332233 & 0 & 0 & 136 & 1533 & 7045 & 5261 & 6025 \\
\hline 22323323 & 0 & 0 & 303 & 2090 & 6014 & 7690 & 3903 \\
\hline 22323233 & 0 & 37 & 1944 & 4367 & 6160 & 4634 & 2858 \\
\hline 22232333 & 0 & 195 & 1476 & 1776 & 3628 & 4546 & 8379 \\
\hline 23232323 & 251 & 929 & 5740 & 3168 & 5420 & 2828 & 1662 \\
\hline
\end{tabular}

random subsets of 8 numbers between 1 and 80, chosen using Maple's random number generator. For each choice, we considered the 8 possibilities of orderings of the indices, and for each system we computed the number of real solutions out of 12 . Observe that the apparent lower bound on the number of real solutions depends on ordering of the indices of the Schubert data. Other calculations we have done reinforce this observation.

We conclude by reminding the reader of the observation in Section 3 that the number of real solutions to a problem in enumerative goemetry depends subtly on the configuration of the conditions.

\section{Acknowledgements}

We thank the following people: Ricky Pollack, Marie-Françoise Roy, Bernd Sturmfels, Andrei Gabrielov, William Fulton, Felice Ronga, Viatcheslav Kharlamov, Thorsten Theobald, and T.-Y. Li.

\section{References}

[1] D. N. Bernstein, The number of roots of a system of equations, Funct. Anal. Appl., 9 (1975), pp. $183-185$.

[2] I. N. Bernstein, I. M. Gelfand, And S. I. Gelfand, Schubert cells and cohomology of the spaces $G / P$, Russian Mathematical Surveys, 28 (1973), pp. 1-26.

[3] A. Bertram, Quantum Schubert calculus, Adv. Math., 128 (1997), pp. 289-305.

[4] R. BRicard, Mémoire sur la théorie de l'octaèdre articulé, J. Math. Pures et Appliquées, 3 (1897), pp. $113-150$.

[5] P. Burgisser, M. Clausen, and M. Shokrollahi, Algebraic Complexity Theory, SpringerVerlag, 1997.

[6] P. Candelas, X. C. de la Ossa, P. S. Green, and L. Parkes, A pair of Calabi-Yau manifolds as an exactly soluble superconformal theory, Nuclear Phys. B, 359 (1991), pp. 21-74.

[7] G. Castelnuovo, Numero delle involuzioni razionali gaicenti sopra una curva di dato genere, Rendi. R. Accad. Lineci, 4 (1889), pp. 130-133.

[8] R. Chinvacci and J. Escamilla-Castillo, Schubert calculus and enumerative problems, Bollettino Un. Math. Ital., 7 (1988), pp. 119-126.

[9] J. Clark, The consistent selection of local coordinates in linear system identification, in Proc. Joint Automatic Control Conference, 1976, pp. 576-580.

[10] D. A. Cox And S. Katz, Mirror symmetry and algebraic geometry, American Mathematical Society, Providence, RI, 1999.

[11] H. S. M. Coxeter, The twenty-seven lines on the cubic surface, in Convexity and its applications, Birkhäuser, Basel, 1983, pp. 111-119.

[12] A. I. Degtyarev and V. M. Kharlamov, Topological properties of real algebraic varieties: Rokhlin's way, Uspekhi Mat. Nauk, 55 (2000), pp. 129-212. 
[13] M. Demazure, Désingularization des variétés de Schubert généralisées, Ann. Sc. E. N. S. (4), 7 (1974), pp. 53-88.

[14] P. Dietmaier, The Stewart-Gough platform of general geometry can have 40 real postures, in Advances in Robot Kinematics: Analysis and Control, Kluwer Academic Publishers, 1998, pp. 1-10.

[15] D. Eisenbud AND J. HARRis, Divisors on general curves and cuspidal rational curves, Invent. Math., 74 (1983), pp. 371-418.

[16] A. Eremenko and A. Gabrielov, Rational functions with real critical points and B. and M. Shapiro conjecture in real enumerative geometry. Mss., January 6, 2000.

[17] —, Degrees of real Grassmann varieties. Mss., March 26, 2001.

[18] — N N New counterexamples to pole placement by static output feedback. Linear Algebra and its Applications, to appear, 2001.

[19] —, Wronski map and real Schubert calculus. Mss., February 5, 2001.

[20] W. Fulton, Intersection Theory, no. 2 in Ergebnisse der Math., Springer-Verlag, 1984.

[21] _ _ Introduction to Intersection Theory in Algebraic Geometry, CBMS 54, AMS, 1996. second edition.

[22] W. Fulton and R. Pandharipande, Notes on stable maps and quantum cohomology. in Algebraic Geometry, Santa Cruz, 1995, J. Kollár, ed., vol. 62, Part 2 of Proc. Sympos. Pure Math., Amer. Math. Soc., 1997, pp. 45-96.

[23] G. Giambelli, Rizoluzione del problema degli spazi secanti, Memor. Real. Acc. di Torino, 52 (1902), pp. 171-193.

[24] L. Goldberg, Catalan numbers and branched coverings by the Riemann sphere, Adv. Math., 85 (1991), pp. 129-144.

[25] V. Gough, Contribution to discussion papers on research in automobile stability and control in tyre performance, 1957.

[26] B. HaAs, A counterexample to Kouchnirenko's conjecture. Beitr. Alg. Geom., 43, to appear, 2002.

[27] A. HARNACK, Über die Vieltheiligkeit der eben algebraischen Kurven, Math. Ann., 10 (1876), pp. $189-198$.

[28] J. HARRIs, Galois groups of enumerative problems, Duke Math. J., 46 (1979), pp. 685-724.

[29] D. HiLbert, Über die Darstellung definiter Formen als Summe von Formen-quadraten, Math. Ann., 32 (1888), pp. $342-350$.

[30] —, Über die reellen Züge algebraischen Curven, Math. Ann., 38 (1891), pp. 115-138.

[31] _ Sur les problèmes futurs des mathématiques, in Proceedings of the Second International Congress of Mathematicians, Paris 1900, Gauthier-Villars, 1902, pp. 58-114.

[32] B. Huber, F. Sottile, And B. Sturmfels, Numerical Schubert calculus, J. Symb. Comp., 26 (1998), pp. 767-788.

[33] B. Huber And B. Sturmfels, A polyhedral method for solving sparse polynomial systems, Math. Comp., 64 (1995), pp. 1541-1555.

[34] B. Huber and J. Verschelde, Pieri homotopies for problems in enumerative geometry applied to pole placement in linear systems control, SIAM J. Control and Optim., 38 (2000), pp. 1265-1287.

[35] J. Huisman, On the number of real hypersurfaces hypertangent to a given real space curve. Mss., 2001.

[36] K. Intriligator, Fusion residues, Mod. Phys. Lett. A, 6 (1991), pp. 3543-3556.

[37] I. Itenberg And M.-F. Roy, Multivariate Descartes' rule, Beiträge zur Algebra und Geometrie, 37 (1996), pp. 337-346.

[38] S. Katz, On the finiteness of rational curves on quintic threefolds, Compositio Math., 60 (1986), pp. 151-162.

[39] V. Kharlamov and F. Sottile, Maximally inflected real rational curves. In preparation, 2001.

[40] A. Khovanskir, Newton polyhedra and the genus of complete intersections, Funct. Anal. Appl., 12 (1978), pp. 38-46.

[41] - Fewnomials, Trans. of Math. Monographs, 88, AMS, 1991.

[42] S. L. Kleiman, The transversality of a general translate, Compositio Math., 28 (1974), pp. 287-297.

[43] — Problem 15. Rigorous foundation of Schubert's enumerative calculus, in Mathematical Developments arising from Hilbert Problems, vol. 28 of Proc. Sympos. Pure Math., Amer. Math. Soc., 1976, pp. 445-482. 
[44] F. KLEIN, Eine neue Relation zwischen den Singularitäten einer algebraischen Kurve, Math. Ann., 10 (1876), pp. 199-209.

[45] A. Kouchnirenko, A Newton polyhedron and the number of solutions of a system of $k$ equations in $k$ unknowns, Usp. Math. Nauk., 30 (1975), pp. 266-267.

[46] L. Kronecker, Leopold Kronecker's Werke, Chelsea, NY, 1968.

[47] D. Larman. Problem posed in the Problem Session of the DIMACS Workshop on Arrangements, Rutgers University, New Brunswick, NJ, USA, 1990.

[48] D. LAZARD, Generalized Stewart platform: how to compute with rigid motions?, in IMACS-SC'93, 1993.

[49] T. Y. Li, J. M. RoJas, And X. Wang, Trinomials in the plane. In preperation, 2001.

[50] T. Y. Li AND X. WAng, On multivariate Descartes' rule-a counterexample, Beiträge Algebra Geom., 39 (1998), pp. 1-5.

[51] I. Macdonald, J. Pach, and T. Theobald, Common tangents to four unit balls in $\mathbb{R}^{3}$. To appear in Discrete and Computational Geometry 26:1 (2001).

[52] G. MegYesi, Lines tangent to 4 spheres with affinely dependent centres. To appear in Discrete and Computational Geometry.

[53] B. MourRain, The 40 generic positions of a parallel robot, in ISSAC'93, Kiev, ACM Press, 1993, pp. $173-182$.

[54] _ Enumeration problems in geometry, robotics, and vision, in Algorithms in Algebraic Geometry and Applications (MEGA-94, Santander), L. González-Vega and T. Recio, eds., vol. 143 of Progress in Math., Birkhäuser, 1996, pp. 285-306.

[55] D. Napoletani, A power function approach to Kouchnirenko's conjceture. Proceedings of Mt. Holyoke conference, 2001.

[56] P. Pedersen and B. Sturmfels, Mixed monomial bases, in Algorithms in Algebraic Geometry and Applications, L. González-Vega and T. Recio, eds., vol. 143 of Progress in Mathematics, Proceedings, MEGA-94, Birkhäuser, 1996, pp. 307-316.

[57] M. PieRI, Sul problema degli spazi secanti, Rend. Reale Ist. lombardo, 26 (1893), pp. 534-546.

[58] V. Powers And B. ReZnick, Notes towards a constructive proof of Hilbert's theorem on ternary quartics, in Quadratic forms and their applications (Dublin, 1999), Amer. Math. Soc., Providence, RI, 2000, pp. 209-227.

[59] M. Raghavan, The Stewart platform of general geometry has 40 configurations, in ASME Design and Automation Conf., vol. 32-2, 1991, pp. 397-402.

[60] M. Ravi, J. Rosenthal, And X. Wang, Degree of the generalized Plücker embedding of a quot scheme and quantum cohomology, Math. Ann., 311 (1998), pp. 11-26.

[61] F. Ronga, A. Tognoli, And T. Vust, The number of conics tangent to 5 given conics: the real case, Rev. Mat. Univ. Complut. Madrid, 10 (1997), pp. 391-421.

[62] F. Ronga And T. Vust, Stewart platforms without computer?, in Real Analytic and Algebraic Geometry, Proceedings of the International Conference, (Trento, 1992), Walter de Gruyter, 1995, pp. $196-212$.

[63] H. Schubert, Kalkul der abzählenden Geometrie, Springer-Verlag, 1879. reprinted with an introduction by S. Kleiman, 1979.

[64] _ _ Anzahl-Bestimmungen für lineare Räume beliebiger Dimension, Acta. Math., 8 (1886), pp. 97118.

[65] — Die n-dimensionalen Verallgemeinerungen der fundamentalen Anzahlen unseres Raume, Math. Ann., 26 (1886), pp. 26-51. (dated 1884).

[66] — L Losüng des Charakteritiken-Problems für lineare Räume beliebiger Dimension, Mittheil. Math. Ges. Hamburg, (1886), pp. 135-155. (dated 1885).

[67] B. Siebert and G. Tian, On quantum cohomology rings of Fano manifolds and a formula of Vafa and Intrilligator, Asian J. Math., 1 (1997), pp. 679-695.

[68] F. SotriLE, Enumerative geometry for real varieties, in Algebraic Geometry, Santa Cruz 1995, J. Kollár, R. Lazarsfeld, and D. Morrison, eds., vol. 62, Part 1 of Proc. Sympos. Pure Math., Amer. Math. Soc., 1997, pp. 435-447.

[69] — Enumerative geometry for the real Grassmannian of lines in projective space, Duke Math. J., 87 (1997), pp. 59-85. 
[70] _ Real enumerative geometry and effective algebraic equivalence, J. Pure Appl. Alg., 117 \& 118 (1997), pp. 601-615. Proc., MEGA'96.

[71] - The conjecture of Shapiro and Shapiro. An archive of computations and computer algebra scripts, http://www .expmath.org/extra/9.2/sottile, 1999.

[72] — The special Schubert calculus is real, ERA of the AMS, 5 (1999), pp. 35-39.

[73] —, Real rational curves in Grassmannians, J. Amer. Math. Soc., 13 (2000), pp. 333-341.

[74] _- Real Schubert calculus: Polynomial systems and a conjecture of Shapiro and Shapiro, Exper. Math., 9 (2000), pp. 161-182.

[75] — Some real and unreal enumerative geometry for flag manifolds, Mich. Math. J., 48 (2000), pp. 573-592. Special Issue in Honor of Wm. Fulton.

[76] — Elementary transversality in the Schubert calculus for arbitrary characteristic. www. arXiv.org/math. AG/0010319, 2001.

[77] _ Rational curves in Grassmannians: systems theory, reality, and transversality. to Appear in 'Enumerative Geometry in Physics', ed. by E. Previato, 2001.

[78] _ Some real and unreal conjectures on enumerative geometry for flag manifolds. In preperation, 2001.

[79] F. Sottile And T. Theobald, Common tangent lines to $2 n-2$ spheres in $\mathbb{R}^{n}$. www . arXiv.org/math. AG/0105180, 2001.

[80] D. Stewart, A platform with 6 degree of freedom, Proc. of the Institution of Mechanical Engineers, 180 (1965-66), pp. 371-386.

[81] B. Sturmfels, On the number of real roots of a sparse polynomial system, in Hamiltonian and gradient flows, algorithms and control, vol. 3 of Fields Inst. Commun., American Mathematical Society, Providence, 1994, pp. 137-143.

[82] _ - Viro's theorem for complete intersections, Ann. della Scuola Nor. Sup. Pisa, Sc. Fis. Mat., Ser IV, XXI (1994), pp. 377-386.

[83] C. VAfa, Topological mirrors and quantum rings, in Essays on Mirror Manifolds, S.-T. Yau, ed., International Press, Hong Kong, 1992, pp. 96-119.

[84] J. Verschelde, Polyhedral homotopies for dense, sparse, and determinantal systems. MSRI preprint 1999-041, 1999.

[85] O. Viro, Real algebraic plane curves: Constructions with controlled topology, Algebra i Analiz, 1 (1989), pp. 1-73. Translation in Leningrad Math. J., 1 (1990), pp. 1059-1134.

[86] C. Wall, Is every quartic a conic of conics?, Math. Proc. Cambridge Philos. Soc., 109 (1991), pp. 419-424.

[87] D. White, Sign-balanced posets. Preprint, Feb. 11, 2000 at white@math.umn.edu.

[88] H. G. Zeuthen, Almindelige Egenskaber ved Systemer af plane Kurver, Danske Videnskabernes Selskabs Skrifter, Naturvidenskabelig og Mathematisk, Afd. 10 Bd. IV, (1873), pp. 286-393.

[89] _ - Sur les différentes formes des courbes planes du quartriéme order, Math. Ann., 7 (1874), pp. $410-432$.

Department of Mathematics, University of Massachusetts, Lederle Graduate ReSearch Tower, Amherst, Massachusetts, 01003, USA

E-mail address: sottile@math.umass.edu

$U R L:$ http://www . math. umass.edu/ ${ }^{\text {sottile }}$ 